\title{
Theoretical Backgrounds of Durability Analysis by Normalized Equivalent Stress Functionals
}

\author{
S.E. Mikhailov \\ Dept. of Mathematics, Glasgow Caledonian Univ., \\ Cowcaddens Road, Glasgow, G4 0BA, UK \\ (s.mikhailov@gcal.ac.uk)
}

\begin{abstract}
Generalized durability diagrams and their properties are considered for a material under a multiaxial loading given by an arbitrary function of time. Material strength and durability under such loading is described in terms of durability, safety factor, and normalized equivalent stress. Relations between these functionals are analysed. Some material properties including time and load stability, self-degradation (aging), monotonous damaging are discussed. Phenomenological strength conditions are presented in terms of the normalized equivalent stress. It is shown that the damage based durability analysis is reduced to a particular case of such strength conditions. Examples of the reduction are presented for some known durability models. The approach is applicable to the strength and durability description at creep and impact loading and their combination.
\end{abstract}

Keywords: Durability; Strength conditions; Endurance limit; Dynamic failure

\section{Introduction}

Different forms of durability description are commonly used for time or history dependent materials possessing plasticity, creep and/or serving under fatigue or impact loadings. A usual auxiliary means for this is introduction of a damage measure and an evolution law for this measure, see, e.g., [1]-[7] and also some remarks in Appendix A. Together with the limiting damage value, which when reached means rupture, this gives a strength condition. Such damage measure is often associated with a geometrical change, that is, with the defect cross-section fraction or the defect volume fraction in a representative volume 
element or with the stiffness change of the damaged material or it is taken as an abstract internal material parameter. The geometrical damage measures involve difficulties in their experimental evaluation, the stiffness damage measure is not always representative, e.g., for high cyclic fatigue. For abstract damage measures, a value of the measure below the critical one delivers no direct information about the safety or the residual life durability. Different abstract measures and their evolution laws are not easy to compare. In addition, most evolution laws do not take into account a dependence of the damage measure rate on the process history. We remark that such a dependence is considered in [5].

In this paper, we will try to show that the phenomenological durability description and analysis can be done completely without such additional means as a damage measure if the loading process $\sigma_{i j}(\tau)$ is known. Note however that a damage analysis can be useful for prediction of the loading process $\sigma_{i j}(\tau, x)$ at each point $x$, particularly for softening materials. Some damage measures can be also quite helpful for obtaining a phenomenological durability description from micro-mechanical considerations.

We shall discuss here a material under a uniform multiaxial stress state. Using some ideas of [8], durability, safety factor and normalized equivalent stress (load factor) are presented in this paper, which are mechanically meaningful and experimentally measurable on the one hand and accumulate process history on the other hand.

Generally, durability analysis includes the following main items: (i) determination of the durability $t^{*}(\sigma)$ for a prescribed loading process $\sigma_{i j}(\tau)$; (ii) determination of the safety factor $\underline{\lambda}(\sigma ; t)$ at an instant $t$ for a prescribed process $\sigma_{i j}(\tau)$; (iii) determination of a damage $\omega(\sigma ; t)$ at an instant $t$ for a prescribed process $\sigma_{i j}(\tau)$; (iv) interpolation of the functionals $t^{*}(\sigma), \underline{\lambda}(\sigma ; t)$, and $\omega(\sigma ; t)$ along their values for some processes $\sigma_{i j}^{r}(\tau), r=1 \ldots R$. We call $t^{*}(\sigma), \underline{\lambda}(\sigma ; t), \omega(\sigma ; t)$ functionals since each of them maps a function $\sigma_{i j}(\tau)$ into a number. Note that although a damage measure is mentioned between the main items, it can be considered as an auxiliary parameter, helping in some models to determine the practically interesting parameters $t^{*}(\sigma)$ and $\underline{\lambda}(\sigma ; t)$. This paper is devoted chiefly to a discussion of definitions, properties and mutual connections of the functionals $t^{*}(\sigma), \underline{\lambda}(\sigma ; t)$, and of the normalized equivalent stress functional $\underline{\Lambda}(\sigma ; t)=1 / \underline{\lambda}(\sigma ; t)$. It develops the results of Mikhailov (1999).

\section{Generalized durability diagram}

\subsection{Durability and strength stability in time}

Let a material undergo a loading program (process) $\sigma_{i j}(\tau)$. We will discuss here rupture without specifying the rupture type and only assume that (i) one can unambiguously detect at any time instant whether the body is ruptured or not, and (ii) if the body is ruptured at an instant $t$, it will be ruptured also at any instant $t_{1}>t$ (no repairing 
mechanism). We say that strength is stable in time (or $t$-stable) at an instant $t$ under a loading process $\sigma_{i j}(\tau)$ if there is no rupture at $t$ and there exists an instant $t^{\prime}(\sigma ; t)>t$ where also no rupture occurs. (This means that the time interval, where strength is stable, is open.) From this definition, strength is unstable in time (or t-unstable) at an instant $t$ under a loading process $\sigma_{i j}(\tau)$ if there is no rupture at $t$ but the body is ruptured at any instant $t^{\prime}(\sigma ; t)>t$ (an example is given by the discontinuous loading process presented on Fig. 5a at $t=\tau^{*}$ if $\sigma_{1}<\sigma_{r} \leq \sigma_{2}$, see below).

If the strength is $t$-stable at all instants where no rupture appears, then the time $t^{*}(\sigma)$, at which a rupture for the material appears is called durability or life time. If there exists an instant where the strength is $t$-unstable, we implement a more general definition of the durability as an instant $t^{*}(\sigma)$ such that there is no rupture at any $t<t^{*}(\sigma)$ and the body is ruptured at any $t>t^{*}(\sigma)$; if there is no rupture at any time $t<\infty$, we say the durability is infinite, $t^{*}(\sigma)=\infty$.

Thus the $t<t^{*}(\sigma)$ is condition of $t$-stable strength at the instant $t$. On the other hand, the equality $t=t^{*}(\sigma)<\infty$ means rupture or strength $t$-instability at the instant $t$.

The life time seems to be the main relevant measurable parameter in the durability analysis and all other parameters are derived from it. For different loading processes $\sigma_{i j}^{1}(\tau), \sigma_{i j}^{2}(\tau)$, the durability has different values $t^{*}\left(\sigma^{1}\right), t^{*}\left(\sigma^{2}\right)$ (see Fig. 1).

\subsection{Durability diagrams}

Let $H(\tau)=\left\{\begin{array}{cc}0, & \tau \leq 0 \\ 1, & \tau>0\end{array}\right\}$ be the Heaviside step-like function. Under a uniaxial steplike loading $\sigma(\tau)=H(\tau) \sigma^{0}$, where $\sigma^{0}$ is a constant, it is usual to determine experimentally the durability diagram in the axes $\sigma^{0} \mapsto t^{*}\left(\sigma^{0}\right)$. Its counterpart in fatigue under a constant stress range oscillation $\Delta \sigma^{0}=\sigma_{\max }^{0}-\sigma_{\min }^{0}$ is the Wöhler diagram $\Delta \sigma^{0} \mapsto n^{*}\left(\Delta \sigma^{0}\right)$, where $n^{*}\left(\Delta \sigma^{0}\right)$ is the number of cycles before rupture.

An example of a simple durability diagram given by a power law (a straight line in the double logarithmic coordinates) can be written as

$$
t^{*}\left(\sigma^{0}\right)=A\left|\sigma^{0}\right|^{-b}
$$

where $A$ and $b$ are positive constants depending on the stress state type (tension, compression or shear). A similar power dependence for a constant in time multiaxial loading $\sigma_{i j}^{0}=$ const. can be written in the form

$$
t^{*}\left(\sigma^{0}\right)=\left|\sigma^{0}\right|^{-b\left(\tilde{\sigma}^{0}\right)} A\left(\tilde{\sigma}^{0}\right) .
$$

Here $\left|\sigma^{0}\right|$ is a matrix norm of the tensor $\sigma_{i j}^{0}$, for example, $\left|\sigma^{0}\right|=\sqrt{\sum_{i, j=1}^{3} \sigma_{i j}^{0} \sigma_{i j}^{0}} ; \tilde{\sigma}_{i j}^{0}=$ $\sigma_{i j}^{0} /\left|\sigma^{0}\right|$ is the normalized stress tensor, presenting the tensor $\sigma_{i j}^{0}$ shape; $A\left(\tilde{\sigma}^{0}\right)$ and $b\left(\tilde{\sigma}^{0}\right)$ are positive parameters depending on the tensor $\sigma_{i j}^{0}$ shape but not on the tensor norm. 
To present a generalised diagram for a multiaxial process described by an arbitrary tensor function $\sigma_{i j}(\tau)$, let us consider a family of proportional processes $\lambda \sigma_{i j}(\tau)$, obtained from the original process $\sigma_{i j}(\tau)$ after its multiplication by a non-negative constant number $\lambda$, Fig. 2.

The generalised durability diagram for a process $\sigma_{i j}(\tau)$ is the dependence of the durability $t^{*}(\lambda \sigma)$ on a parameter $\lambda \geq 0$.

The concept propounded in the paper concerns mainly time and history dependent materials but should work also in the particular case of materials independent of time and history. We will extensively use the latter for illustrations.

Let us consider, for example, a material independent of time and history, uniaxially loaded by a step-like process $\sigma(\tau)=H(\tau) \sigma^{0}$, where $\sigma^{0}$ is a constant, and obeying the strength condition $\sigma<\sigma_{r}$, where $\sigma_{r}$ is constant. Then the durability diagram is given by the line $\lambda=\sigma_{r} / \sigma^{0}$, that is, $t^{*}(\lambda \sigma)=\left\{\begin{array}{cc}\infty, & \lambda<\sigma_{r} / \sigma^{0} \\ 0, & \lambda \geq \sigma_{r} / \sigma^{0}\end{array}\right\}$.

If the loading process for the same material is $\sigma(\tau)=a \tau$, where $a$ is a constant, then the durability diagram is a hyperbola $t^{*}(\lambda \sigma)=\sigma_{r} /(a \lambda)$.

Let us consider an arbitrary material. Suppose $\sigma_{i j}(\tau)$ is a multiaxial step-like process $\sigma_{i j}(\tau)=H(\tau) \sigma_{i j}^{0}$, where $\sigma_{i j}^{0}$ is a constant tensor, $\left|\sigma^{0}\right|=1$. It is evident, that the generalised durability diagram $\lambda \mapsto t^{*}(\lambda \sigma)$ coincides with the classical durability diagram $|\sigma| \mapsto t^{*}(\sigma)$ for the step-like processes $\sigma_{i j}(\tau)=|\sigma| H(\tau) \sigma_{i j}^{0}$.

Similarly, the generalised durability diagram $\lambda \mapsto t^{*}(\lambda \sigma)$ for a periodic loading process $\left\{\sigma_{i j}(\tau)=H(\tau) f(\tau) \sigma_{i j}^{0}\right.$, where $\sigma_{i j}^{0}=$ const., $\left|\sigma^{0}\right|=1, f(\tau)$ is a $t_{0}$-periodic function with the unit range, $\left.\Delta f=f_{\max }-f_{\min }=1\right\}$, coincides with the classical Wöhler diagram $\Delta \sigma \mapsto n^{*}(\Delta \sigma)$ for the oscillating processes $\sigma_{i j}(\tau)=\Delta \sigma H(\tau) f(\tau) \sigma_{i j}^{0}$, where $n^{*}=t^{*} / t_{0}$.

Let us consider general properties of the generalised durability diagram $t^{*}(\lambda \sigma)$ for an arbitrary material under a given process $\sigma_{i j}(\tau)$. This function is defined on the half axis $\lambda \in[0, \infty)$ and is non-negative. When $\lambda$ varies, different situations can arise. We plot schematically a durability diagram on Fig. 3a. Although we consider $t^{*}(\lambda \sigma)$ as a function of $\lambda$, the choice of the axes directions on the plot is traditional for the durability analysis. The curves $a, b, c$ at large $\lambda$ and curves $d, e, f$ at small $\lambda$ present different possible cases of the diagram behaviour, that is, one of the curves $a, b$ or $c$ continues by one of the curves $d, e$ or $f$ for a particular material under a particular loading $\sigma_{i j}(\tau)$.

We analyse first small durabilities $t^{*}$, that is, large $\lambda$.

(A): The rupture can occur at $t=t^{*}\left(\lambda^{0} \sigma\right)=0$ for a finite but sufficiently large $\lambda^{0}$, curve $a$ on Fig. 3a. It can happen, for example, for some materials under the step-like loading $\sigma_{i j}(\tau)=H(\tau) \sigma_{i j}^{0}$ where $\sigma_{i j}^{0}$ is a constant tensor. Particularly, as mentioned above, this diagram is the horizontal line $\lambda=\lambda^{0}=\sigma_{r} / \sigma^{0}$ for a time and history independent material under such loading.

(B): The durability $t^{*}\left(\lambda \sigma_{i j}\right)$ can be non-zero at any finite $\lambda$ but tends to zero as $\lambda$ 
tends to infinity, curve $b$ on the Fig. 3 a; then $\lambda^{0}=\infty$. Particularly, this is the case for a loading process growing continuously from zero, e.g. for $\sigma_{i j}(\tau)=\tau \sigma_{i j}^{0}$ where $\sigma_{i j}^{0}$ is a constant tensor. This is the case also under the step-like loading $\sigma_{i j}(\tau)=H(\tau) \sigma_{i j}^{0}$ for materials obeying some dynamic strength conditions, see e.g. Sections 6.2, 6.3.

(C): There exist loadings for some materials (or material models), that do not cause rupture however large these loadings are. An example is the uniform three-axes compression, $\sigma_{i j}=\delta_{i j}$. Suppose a loading process $\sigma_{i j}(\tau)$ is represented by such a loading on a beginning time interval $0 \leq \tau \leq t_{+}$followed by a loading able to cause rupture at some time. Then there is no rupture on $0 \leq \tau \leq t_{+}$for any non-negative $\lambda$, curve $c$ on the Fig. 3a, and we can put $\lambda=\lambda^{0}=\infty$ on this segment.

Let us consider the durability behaviour at large $t^{*}$, that is, at small $\lambda$.

Let $\lambda=0$. The durability $t^{*}(0)$, when no loading is applied, is either finite or infinite.

(0): The first case means that rupture at $t=t^{*}(0)<\infty$ is caused not by a mechanical load $\sigma_{i j}(\tau), \tau \geq 0$ but for another reason, for example, by a previous loading history at $\tau<0$. Other possible reasons for such behaviour can be radiation, corrosion or other chemical reactions, dissolution etc., which we can refer to as natural or artificial ageing leading to the complete degradation of the material at the time $t^{*}(0)$. We call the material self-degrading if $t^{*}(0)<\infty$.

Note that ageing does not necessarily lead to complete degradation. Generally, $a$ material is said to be ageing in strength if $t^{*}\left(\sigma^{\Delta}\right) \neq t^{*}(\sigma)+\Delta$, where $\sigma_{i j}^{\Delta}(\tau)=\sigma_{i j}(\tau+\Delta)$, for some $\sigma_{i j}(\tau)$ and $\Delta$. This means, a shift of a loading process in time does not cause the same shift in durability for an ageing material.

Return to the description of Fig.3a.

(D): The durability $t^{*}(\lambda \sigma)$ tends to a finite value $t^{* 0}(\sigma) \leq t^{*}(0)$ as $\lambda$ tends to 0 , curve $d$ on Fig. 3a. Usually one can expect continuity, i.e. $t^{* 0}(\sigma)=t^{*}(0)$ but it is not always the case since $t^{* 0}(\sigma)$, unlike $t^{*}(0)$, is determined not only by the material properties but also by loading. For example, $t^{* 0}(\sigma)<t^{*}(0)$ for a singular stress $\sigma_{i j}(\tau)$ infinitely growing as $\tau$ tends to $t^{* 0}$, i.g., for $\sigma_{i j}(\tau)=\sigma_{i j}^{0} /\left(t^{* 0}-\tau\right)$. Obviously, $t^{* 0}(\sigma)$ can be finite also for non-self-degrading materials, i.e. for $t^{*}(0)=\infty$.

If $t^{*}(0)=\infty$, that is, the material is not self-degrading, we can have three possible situations.

$(\mathrm{E}): t^{*}(\lambda \sigma) \rightarrow t^{* 0}(\sigma)=\infty$ as $\lambda \rightarrow 0$ and there exists no non-zero threshold, that is, the durability $t^{*}(\lambda \sigma)$ monotonously grows up to infinity with diminishing $\lambda$ but is always finite at $\lambda>0$, curve $e$ on Fig. 3a.

$(\mathrm{F}): t^{*}(\lambda \sigma) \rightarrow t^{* 0}(\sigma)=\infty$ as $\lambda \rightarrow 0$ and there exists a threshold value $\lambda_{t h}(\sigma)>0$ such that $t^{*}(\lambda \sigma)=\infty$ for all $\lambda$ such as $0 \leq \lambda \leq \lambda_{t h}(\sigma)$ and $t^{*}(\lambda \sigma)<\infty$ for all $\lambda>\lambda_{t h}(\sigma)$, curve $f$ on Fig. 3a.

$(\mathrm{G}): t^{*}(\lambda \sigma)$ has no definite limit $t^{* 0}(\sigma)$, this means it is not monotonous as $\lambda \rightarrow 0$. This can happen for materials and processes that are not monotonously damaging (see 
below).

Cases $\mathrm{E}$ and $\mathrm{F}$ seem to be most usual in the durability analysis.

Let us analyse the durability diagram for intermediate $\lambda$.

First, the dependence $t^{*}(\lambda \sigma)$ on $\lambda$ can be either monotonously non-increasing or not. In the former case, that is, if $t^{*}\left(\lambda_{1} \sigma\right) \geq t^{*}\left(\lambda_{2} \sigma\right)$ for any numbers $\lambda_{2}>\lambda_{1} \geq 0$, the process will be called monotonously damaging (MD). A material is monotonously damaging if all processes are monotonously damaging for it.

Note that there exist materials that are not monotonously damaging. For example, strength and durability of solidifying or cemented materials can be essentially increased, if the contracting loading is increased during the solidification or cementation phase, see Fig. 4.

Second, the durability diagrams can have finite jumps along $\lambda$ as well as along $t^{*}(\lambda \sigma)$ axes. Fig. 5, 6, and 7 give some examples of such loading processes for a material independent of time and history, in which rupture appears at $\sigma=\sigma_{r}$.

\subsection{Strength stability in proportional load perturbations}

Strength is said to be stable with respect to proportional load perturbations ( $\lambda$-stable) under a process $\sigma_{i j}(\tau)$ at an instant $t<\infty$, if there is no rupture at and before the instant $t$ under $\sigma_{i j}(\tau)$ and under slightly higher or lower loading. More precisely, there exists $\epsilon>0$ such that there is no rupture at and before the instant $t$ under the process $\lambda \sigma_{i j}(\tau)$ for any $\lambda \in(1-\epsilon, 1+\epsilon)$.

This implies that if the strength in a body is $\lambda$-unstable at an instant $t_{1}$, it can not become $\lambda$-stable at any instant $t_{2}>t_{1}$.

We will denote by $t_{s t}^{*}(\sigma)$ the critical time, that is such that strength is $\lambda$-stable at all instants $t<t_{s t}^{*}(\sigma)$ but either rupture or strength $\lambda$-instability exists at all $t>t_{s t}^{*}(\sigma)$. If strength is $\lambda$-stable at all instants $t<\infty$, we take $t_{s t}^{*}(\sigma)=\infty$.

It is evident, that the critical time $t_{s t}^{*}(\sigma)$ is not greater than the durability $t^{*}(\sigma)$ and the strength is not only $\lambda$-stable but also $t$-stable at $t<t_{s t}^{*}(\sigma)$. If $t_{s t}^{*}(\sigma)=t^{*}(\sigma)$, then either rupture exists or strength is $t$-unstable and $\lambda$-(stable or unstable), at time $t=t_{s t}^{*}(\sigma)$. If $t_{s t}^{*}(\sigma)<t^{*}(\sigma)$, then strength is $t$-stable and $\lambda$-(stable or unstable) at time $t=t_{s t}^{*}(\sigma)$ but $t$-stable and $\lambda$-unstable at $t \in\left(t_{s t}^{*}(\sigma), t^{*}(\sigma)\right)$. This means, the durability diagram has at $\lambda=1$ a horizontal jump on the half-interval $\left[t_{s t}^{*}(\sigma), t^{*}(\sigma)\right)$, where the diagram has no values (see Fig. $7 \mathrm{~b}$ at $\sigma_{m}=\sigma_{r}$ ).

Strength is said to be absolutely stable ( $t \lambda$-stable) under a process $\sigma_{i j}(\tau)$ at an instant $t$, if strength is $t$-stable at and before the instant $t$ under $\sigma_{i j}(\tau)$ and under a slightly higher or lower loading. More precisely, there exists $\epsilon>0$ such that such that $t<t^{*}(\lambda \sigma)$ for any $\lambda \in(1-\epsilon, 1+\epsilon)$.

Evidently, if strength is absolutely stable at an instant $t$, it is also $t$-stable and 
$\lambda$-stable at the same instant $t$. On the other hand, strength is absolutely stable under a process $\sigma_{i j}(\tau)$ at any instant $t<t_{s t}^{*}(\sigma)$. However, strength can be $t$-stable and $\lambda$-stable but not absolutely stable at $t=t_{s t}^{*}(\sigma)<t^{*}(\sigma)$.

For $t=\infty$, the above reasoning can be modified by the following way.

Endurance is said to be stable with respect to proportional load perturbations ( $\lambda$-stable) under a process $\sigma_{i j}(\tau)$, if there is no rupture under $\sigma_{i j}(\tau)$ and under a slightly higher or lower loading at any time. More precisely, there exists $\epsilon>0$ such that there is no rupture at all time instants $t<\infty$ under the process $\lambda \sigma_{i j}(\tau)$ for any $\lambda \in(1-\epsilon, 1+\epsilon)$.

Evidently, $\lambda$-stable endurance under a process $\sigma_{i j}(\tau)$ imlies $t_{s t}^{*}(\sigma)=\infty$. However, the equality $t_{s t}^{*}(\sigma)=\infty$ does not generally imply $\lambda$-stable endurance.

Returning to the examples description, we note that the discontinuous monotonous process on Fig. 5a generates a continuous durability diagram $\lambda \mapsto t^{*}(\lambda \sigma)$, Fig. 5b, with a finite jump along the $\lambda$ axis. The strength under the process is $\lambda$-stable but $t$-unstable at $\tau=\tau^{*}$ if $\sigma_{1}<\sigma_{r} \leq \sigma_{2} ; t_{s t}^{*}(\sigma)=t^{*}(\sigma)=\tau^{*}$ for this case.

The continuous non-monotonous process on Fig. 6a, generates a discontinuous (rightcontinuous) durability diagram $\lambda \mapsto t^{*}(\lambda \sigma)$, Fig. 6b. If there exists strength at an instant $t$, the strength is absolutely stable. Rupture appears at $t=\tau_{1}$ if $\sigma_{m}=\sigma_{r}$.

The discontinuous (right-continuous) non-monotonous process Fig. 7a generates a discontinuous (left-continuous) durability diagram $\lambda \mapsto t^{*}(\lambda \sigma)$, Fig. 7b. The strength under the process is $t$-stable but $\lambda$-unstable at $t \in\left[\tau_{1}^{*}, \tau_{2}^{*}\right)$ if $\sigma_{m}=\sigma_{r} ; t_{s t}^{*}(\sigma)=\tau_{1}<t^{*}(\sigma)=\tau_{2}^{*}$ for this case. (Recall that one should turn the diagrams on Fig. 6b, 7b appropriately making the axis $\lambda$ horizontal, to interpret the diagrams right-(left-)continuity literally).

Some relations between strength $\lambda$-stability and continuity of the durability diagram are given in Appendix B.

\section{$3 \quad$ Safety factor and normalized equivalent stress}

For a given process $\sigma_{i j}(\tau)$, we can determine (experimentally) a unique finite, infinite, or zero value of durability $t^{*}(\lambda \sigma)$ for any number $\lambda \geq 0$. Consider the inverse task: for any $t \geq 0$, to determine a number $\lambda^{*}(\sigma ; t)$ such that $t^{*}\left(\lambda^{*}(\sigma ; t) \sigma\right)=t$. This is equivalent interpreting the durability diagram $\lambda \mapsto t^{*}(\lambda \sigma)$ as the dependence $t \mapsto \lambda^{*}(\sigma ; t)$. Examples of the diagrams on Fig. $3 \mathrm{a}, 4 \mathrm{~b}, 5 \mathrm{~b}, 6 \mathrm{~b}, 7 \mathrm{~b}$ show this is not always uniquely possible since the dependence is either not defined or not unique for some instants $t$. The following definition concerns the cases when this is possible.

Definition 1CM If the durability $t^{*}(\lambda \sigma)$ is a continuous and monotonously decreasing function of $\lambda$, the temporal safety factor $\underline{\lambda}^{T}(\sigma ; t)$ is the non-negative number, by which the loading process $\sigma_{i j}(\tau)$ must be multiplied to obtain the durability $t$, that is, $t^{*}\left(\underline{\lambda}^{T}(\sigma ; t) \sigma\right)=$ $t$. 
This simple definition of the safety factor is however not applicable if the durability diagram $t^{*}(\lambda \sigma)$ is not a monotonously decreasing function of $\lambda$ as on Fig. 4b and on Fig. 5b at $\tau=\tau^{*}$, since $\lambda^{*}(\sigma ; t)$ then appears to be multiply defined. It is also not applicable if $t^{*}(\lambda \sigma)$ has a horizontal jump as on Fig. 6b since $\lambda^{*}(\sigma ; t)$ appears to be not defined for $\tau_{1}<t<\tau_{2}$.

If $\sigma_{i j}(\tau)$ is an MD process, that is the durability $t^{*}(\lambda \sigma)$ is a monotonously nonincreasing although generally discontinuous function of $\lambda$, we generalise the definition by the following way:

Definition 1MD The temporal safety factor $\underline{\lambda}^{T}(\sigma ; t)$ for a monotonously damaging process $\sigma_{i j}(\tau)$ is supremum of non-negative numbers $\lambda$ such that the durability $t^{*}(\lambda \sigma)$ is greater then $t$; if there is no such $\lambda$, we take $\underline{\lambda}^{T}(\sigma ; t)=0$.

To overcome the difficulties with non-monotonously damaging processes, we introduce the following general definition of the safety factor $\underline{\lambda}(\sigma ; t)$ embracing also the previous particular cases.

Definition 1 The temporal safety factor $\underline{\lambda}^{T}(\sigma ; t)$ is supremum of $\lambda \geq 0$ such that $t^{*}\left(\lambda^{\prime \prime} \sigma\right)>t$ for any $\lambda^{\prime \prime} \in[0, \lambda]$; if there is no such $\lambda$, we take $\underline{\lambda}^{T}(\sigma ; t)=0$. The mapping $(\sigma ; t) \mapsto \underline{\lambda}^{T}(\sigma ; t)$ defined on a set of processes $\sigma_{i j}(\tau)$ and time instants $t$ is called the (strength) safety factor functional $\underline{\lambda}^{T}$.

Note that we can equivalently define $\underline{\lambda}^{T}(\sigma ; t)$ as a non-negative number such that $t^{*}(\lambda \sigma)>t$ for any $\lambda \in\left[0, \underline{\lambda}^{T}(\sigma ; t)\right)$ but for any $\lambda>\underline{\lambda}^{T}(\sigma ; t)$ there exists a number $\lambda^{\prime \prime} \in\left[\underline{\lambda}^{T}(\sigma ; t), \lambda\right]$ such that $t^{*}\left(\lambda^{\prime \prime} \sigma\right) \leq t$; if there is no such $\underline{\lambda}^{T}(\sigma ; t)$, one should take $\underline{\lambda}^{T}(\sigma ; t)=0$.

Definition 2 The temporal normalized equivalent stress $\underline{\Lambda}^{T}(\sigma ; t)$ is defined as $1 / \underline{\lambda}^{T}(\sigma ; t)$; if $\underline{\lambda}^{T}(\sigma ; t)=0$, we take $\underline{\Lambda}^{T}(\sigma ; t)=\infty$.

The mapping $(\sigma ; t) \mapsto \underline{\Lambda}^{T}(\sigma ; t)$ defined on a set of processes $\sigma_{i j}(\tau)$ and time instants $t$ is called the temporal normalized equivalent stress functional (TNESF) $\underline{\Lambda}^{T}$.

From the definition, if the durability $t^{*}(\sigma)$ is known, the value of the TNESF $\underline{\Lambda}^{T}(\sigma ; t)$ is a solution of the scalar equation

$$
t^{*}(\sigma / \Lambda)=t
$$

for each instant $t$ and loading process $\sigma(\tau)$ such that dependence of the durability $t^{*}(\lambda \sigma)$ on $\lambda$ is continuous and monotonous; if $t^{*}(\lambda \sigma)$ is not continuous or not monotonous, $\underline{\Lambda}^{T}(\sigma ; t)$ is given by Definitions $1-2$.

As follows from Definitions 1 and 2, the functionals $\underline{\lambda}^{T}$ and $\underline{\Lambda}^{T}$ do exist for any material with unambiguous detection of strength/rupture status and without repairing mechanism, 
and are unique, that is, are material characteristics for a prescribed environment (temperature, pre-history, etc.).

Remark 1 One can observe from Definitions $1 M D$ and 2 (see Appendix $C$ ) that one can replace the durability $t^{*}(\lambda \sigma)$ by the critical time $t_{s t}^{*}(\lambda \sigma)$ in the definitions to arrive at exactly the same functionals $\underline{\lambda}, \underline{\Lambda}$ for $M D$ processes $\sigma(\tau)$.

The temporal safety factor $\underline{\lambda}^{T}(\sigma ; t)$ and the temporal normalized equivalent stress $\underline{\Lambda}^{T}(\sigma ; t)$ are counterparts of the non-local safety factor $\underline{\lambda}(\sigma ; x)$ and non-local normalized equivalent stress (load factor) $\underline{\Lambda}(\sigma ; x)$ defined in $[8]$

The safety factor and the TNESF introduced by Definitions 1-3 are durability-based. One can introduce also the corresponding strength-based functionals, coinciding with durability-based ones everywhere except the points of their discontinuity in $t$ and we will describe them elsewhere.

For brevity, we will drop the superscript $T$ sometimes further in the paper.

To justify the title normalized equivalent stress for $\underline{\Lambda}$, we consider a constant in time process $\sigma_{i j}(\tau)=$ const. Let, for example, the material strength be associated with the von Mises equivalent stress $\sigma^{e}(\sigma)=\sqrt{\left[\left(\sigma_{1}-\sigma_{2}\right)^{2}+\left(\sigma_{2}-\sigma_{3}\right)^{2}+\left(\sigma_{3}-\sigma_{1}\right)^{2}\right] / 2}$, that is the strength condition has the form $\sigma^{e}(\sigma)<\sigma_{r}(t)$, where the function $\sigma_{r}(t)$ is a material characteristic (classical durability diagram under the uniaxial tension) and $\sigma_{1}, \sigma_{2}, \sigma_{3}$ are the principal stresses. Then $\underline{\Lambda}(\sigma ; t)$ is defined from the equation $\sigma^{e}(\sigma / \Lambda)=\sigma_{r}(t)$, that is

$$
\underline{\Lambda}(\sigma ; t)=\sigma^{e}(\sigma) / \sigma_{r}(t) .
$$

Formula (2) holds true not only for the von Mises equivalent stress but also for the Tresca and any other equivalent stress representations $\sigma^{e}(\sigma)$ that are functions positively homogeneous of the order +1 .

One can see from Definitions 1 and 2 that the safety factor is a non-increasing and the normalized equivalent stress is a non-decreasing function of time, that is,

$$
\underline{\lambda}\left(\sigma ; t_{2}\right) \leq \underline{\lambda}\left(\sigma ; t_{1}\right), \underline{\Lambda}\left(\sigma ; t_{2}\right) \geq \underline{\Lambda}\left(\sigma ; t_{1}\right) \text { if } t_{2}>t_{1} .
$$

Since, suppose the opposite: there exists $t_{2}>t_{1}$ such that $\underline{\lambda}\left(\sigma ; t_{2}\right)>\underline{\lambda}\left(\sigma ; t_{1}\right)$. From $\underline{\lambda}$ definition then there exists $\lambda$ such that $\underline{\lambda}\left(\sigma ; t_{2}\right)>\lambda>\underline{\lambda}\left(\sigma ; t_{1}\right)$ and $t_{2}<t^{*}(\lambda \sigma) \leq t_{1}$. This contradicts to the condition $t_{2}>t_{1}$.

As follows from Definitions 1 and 2 (see Appendix D), for any $t$, the safety factor functional and the TNESF are non-negative positively-homogeneous functionals of the orders -1 and +1 respectively, that is

$$
\underline{\lambda}(k \sigma ; t)=\frac{1}{k} \underline{\lambda}(\sigma ; t) \geq 0, \quad \underline{\Lambda}(k \sigma ; t)=k \underline{\Lambda}(\sigma ; t) \geq 0, \quad \text { for any } k>0 .
$$

For infinite time $t$ we get from here the corresponding definition of the endurance safety factor and temporal endurance normalise equivalent stress 
Definition 3 The temporal endurance (threshold) safety factor $\underline{\lambda}_{t h}^{T}(\sigma)$ is supremum of $\lambda \geq 0$ such that there is no rupture for all $t<\infty$ under the process $\lambda^{\prime \prime} \sigma$ for any $\lambda^{\prime \prime} \in[0, \lambda]$; if there is no such $\lambda$, we take $\underline{\lambda}_{t h}^{T}(\sigma)=0$.

The temporal endurance (threshold) normalized equivalent stress is defined as $\underline{\Lambda}_{t h}^{T}(\sigma)=$ $1 / \underline{\lambda}_{t h}^{T}(\sigma) ;$ if $\underline{\lambda}_{t h}^{T}(\sigma)=0$, we take $\underline{\Lambda}_{t h}^{T}(\sigma)=\infty$.

The mappings $\sigma \mapsto \underline{\lambda}_{t h}^{T}(\sigma), \sigma \mapsto \underline{\Lambda}_{t h}^{T}(\sigma)$ defined on a set of processes $\sigma_{i j}(\tau)$ are called the temporal endurance (threshold) safety factor functional $\underline{\lambda}_{t h}$ and the temporal endurance (threshold) normalized equivalent stress functional $\underline{\Lambda}_{t h}$, respectively.

Owing to monotonicity (3), we can define the endurance functionals also as

$$
\underline{\lambda}_{t h}^{T}(\sigma)=\underline{\lambda}^{T}(\sigma ; \infty):=\inf _{t<\infty} \underline{\lambda}^{T}(\sigma ; t), \quad \underline{\Lambda}_{t h}^{T}(\sigma)=\underline{\Lambda}^{T}(\sigma, \infty):=\sup _{t<\infty} \underline{\Lambda}^{T}(\sigma ; t) .
$$

We can point out the cases, described in the previous section, for which $\underline{\lambda}_{t h}(\sigma)=0$ : case (0) when material is self-degrading, i.e. $t^{*}(0)<\infty$; case (D), i.e. $t^{*}(\lambda \sigma) \rightarrow t^{* 0}(\sigma) \neq$ $\infty$ as $\lambda \rightarrow 0$; case $(\mathrm{E})$; case $(\mathrm{G})$ since the absence of a limit of the function $t^{*}(\lambda \sigma)$ as $\lambda \rightarrow 0$ implies that there exists $t<\infty$ such that $\underline{\lambda}(\sigma ; t)=0$.

Evidently, the endurance safety factor and the endurance normalized equivalent stress make sense as material characteristics only for non-self-degrading materials. As follows from the self-degradation definition above, a material is self-degrading, if and only if there exists an instant $t^{*}(0)$ such that $\underline{\lambda}(0 ; t)=\infty$ for $t<t^{*}(0)$ and $\underline{\lambda}(0 ; t)=0$ for $t \geq t^{*}(0)$. This statement gives an equivalent definition of self-degradation in terms of the safety factor $\underline{\lambda}$ behaviour.

The safety factor $\underline{\lambda}(\sigma ; t)$ as a function of $t$ at a given process $\sigma(\tau)$, can also be considered as a generalised durability diagram $t \mapsto \underline{\lambda}(\sigma ; t)$. It coincides with the monotonous continuous parts of the corresponding diagram $\lambda \mapsto t^{*}(\lambda \sigma)=t$ giving there $\underline{\lambda}\left(\sigma ; t^{*}(\lambda \sigma)\right)=$ $\lambda$, it cuts off the non-monotonous (multi-valued) parts of the diagram $\lambda^{*}(\sigma ; t)$ (taking the branch with the lowest $\lambda^{*}$ and making a corresponding finite jump in $\underline{\lambda}(\sigma ; t)$ in the branch beginning, see Fig. 4) and continues the diagram onto the jump segment $\left[t^{*}((\lambda-0) \sigma), t^{*}((\lambda+0) \sigma)\right]$ where $\lambda^{*}(\sigma ; t)$ does not exist, see Fig. 6, 7. As a result, the diagram looks like a curve on Fig. 3a consisting of corresponding branches with, in addition, possible vertical jumps but without complications like on Fig. 4b, 6b or 7b. As shown above in this section, the diagram is monotonously non-increasing in time. The collection of such diagrams for all possible processes in fact defines the functional $\underline{\lambda}$.

From the generalised durability diagram $t \mapsto \underline{\lambda}(\sigma ; t)$ for a given process $\sigma_{i j}(\tau)$, presented e.g. on Fig. 3a, we can obtain the corresponding diagram $t \mapsto \underline{\Lambda}(\sigma ; t)=1 / \underline{\lambda}(\sigma ; t)$ for the normalized equivalent stress $\underline{\Lambda}(\sigma ; t)$, Fig. 3b. Different curves correspond to different possible cases of its behaviour described in points (A)-(F) of Section 2. Generally, the $t \mapsto \underline{\Lambda}(\sigma ; t)$ diagram can have vertical jumps and is a non-decreasing function of time, see (3). Some examples are given on Fig. (4c), (5c), (6c), (7c). 
The diagram $t \mapsto \underline{\Lambda}(\sigma ; t)$ can be used in two ways. First, it shows a number $\underline{\Lambda}(\sigma ; t)$ such that there is no rupture up to time $t$ for any process $\sigma_{i j}(\tau) / \Lambda^{\prime}$ with $\Lambda^{\prime}>\underline{\Lambda}(\sigma ; t)$. For example, if the diagram includes the curve $f$ (Fig. 3), then the process $\sigma_{i j}(\tau) / \Lambda^{\prime}$ with $\Lambda^{\prime}>\underline{\Lambda}_{t h}(\sigma)$ causes no rupture for any $t$. Another way is to use the diagram together with the stable strength condition (6) below for given $\sigma_{i j}(\tau)$ and $t$. For example, if the diagram includes the curve $f$, then the process $\sigma_{i j}(\tau)$ causes no rupture for any $t$ if $\underline{\Lambda}_{t h}(\sigma)<1$.

\section{Strength and endurance conditions}

Let $\sigma_{i j}(\tau)$ be a process and $t$ be a time instant. The following conclusions can be drown from Definitions 1, 2 for TNESF,

(i) The inequality

$$
\underline{\Lambda}(\sigma ; t)<1
$$

implies absolutely stable strength under the process $\sigma_{i j}(\tau)$ at any instant $\tau \leq t$.

(ii) The equality

$$
\underline{\Lambda}(\sigma ; t)=1
$$

implies

(a) either strength $t$-stable at any $\tau \leq t$ but not absolutely stable at an instant $\tau \leq t$ under the process $\sigma_{i j}(\tau)$, that is, $t^{*}(\sigma)>t$ but for any $\lambda>1$ there exists $\lambda^{\prime \prime} \in(1, \lambda]$ such that $t^{*}\left(\lambda^{\prime \prime} \sigma\right) \leq t$

(b) or rupture (or $t$-unstable strength) under the process $\sigma_{i j}(\tau)$ at an instant $\tau \leq t$, that is, $t^{*}(\sigma) \leq t$.

(iii) If $\sigma_{i j}(\tau)$ is an MD process, the inequality

$$
\underline{\Lambda}(\sigma ; t)>1
$$

implies rupture (or $t$-unstable strength) under the process $\sigma_{i j}(\tau)$ at an instant $\tau \leq t$, that is, $t^{*}(\sigma) \leq t$.

Let us show that, inversely, if strength is absolutely stable for an MD process $\sigma_{i j}(\tau)$ at all $\tau \leq t$ then (6) is satisfied. The strength absolute stability means that there exists $\lambda>1$ such that $t^{*}\left(\lambda^{\prime \prime} \sigma\right)>t$ for any $\lambda^{\prime \prime} \in[1, \lambda]$. In addition, $t^{*}\left(\lambda^{\prime \prime} \sigma\right)>t$ also for all $\lambda^{\prime \prime} \in[0,1]$ since the process $\sigma_{i j}(\tau)$ is monotonously damaging. Application of Definitions 1 and 2 completes the proof of the following statement.

Statement 1 Inequality (6) gives a sufficient (and necessary, if $\sigma_{i j}(\tau)$ is an MD process) condition of absolutely stable strength at all $\tau \leq t$ under the process $\sigma_{i j}(\tau)$.

For the endurance functionals, we similarly have from Definition 3 the following conclusions:

(i) The inequality

$$
\underline{\Lambda}_{t h}(\sigma)<1
$$


implies $\lambda$-stable endurance under the process $\sigma_{i j}(\tau)$.

(ii) The equality

$$
\underline{\Lambda}_{t h}(\sigma)=1
$$

implies

(a) either $\lambda$-unstable endurance, that is, there is no rupture under the process $\sigma_{i j}(\tau)$ at any time but for any $\lambda>1$ there exists $\lambda^{\prime \prime} \in(1, \lambda]$ such that $t^{*}\left(\lambda^{\prime \prime} \sigma\right)<\infty$;

(b) or rupture at an instant $t<\infty$ that is, $t^{*}(\sigma)<\infty$.

(iii) If $\sigma_{i j}(\tau)$ is an MD process, the inequality

$$
\underline{\Lambda}_{t h}(\sigma)>1
$$

implies rupture at an instant $t<\infty$, that is, $t^{*}(\sigma)<\infty$.

Then we have the following statement.

Statement 2 Inequality (9) gives a sufficient (and necessary, if $\sigma_{i j}(\tau)$ is an MD process) condition of $\lambda$-stable endurance under the process $\sigma_{i j}(\tau)$.

Conditions (6)-(11) together with the homogeneity of $\underline{\Lambda}$ and $\underline{\Lambda}_{t h}$ also show that the functionals do really play the role of normalized equivalent stresses.

It follows from the TNESF definition that if the durability diagram $t^{*}(\lambda \sigma)$ is known for a process $\sigma_{i j}(\tau)$ for all $\lambda \geq 0$, then the normalized equivalent stress $\underline{\Lambda}(\sigma ; t)$ can be obtained for $\sigma_{i j}(\tau)$ for any $t \geq 0$. Let us consider an inverse task. Suppose values of the $\operatorname{TNESF} \underline{\Lambda}(\sigma ; t)$ are known for a process $\sigma_{i j}(\tau)$ for any $t \geq 0$. Is it possible to obtain values of the durability diagram $t^{*}(\lambda \sigma)$ for any $\lambda \geq 0$ for the process $\sigma_{i j}(\tau)$ ?

It is evident that this is not possible if $\sigma_{i j}(\tau)$ is not an MD process, since the information about the non-monotonous behaviour of $t^{*}(\lambda \sigma)$ as function of $\lambda$ is lost in $\underline{\Lambda}(\sigma ; t)$. On the other hand if not only inequality (3) hold but $\underline{\Lambda}(\sigma ; t)$ is a monotonously increasing and continuous function of $t$, then it is evident, that $t^{*}(\lambda \sigma)$ is a solution of the following scalar equation

$$
\underline{\Lambda}\left(\sigma ; t^{*}\right)=1 / \lambda
$$

and this solution exists and is unique if $\underline{\Lambda}(\sigma ; 0) \leq 1 / \lambda \leq \underline{\Lambda}(\sigma ; \infty)$.

Note that generally equality (12) can be not satisfied even for arbitrary MD processes but the following inequality holds for any process,

$$
\underline{\Lambda}\left(\sigma ; t^{*}(\lambda \sigma)\right) \geq 1 / \lambda \text { for all } \lambda>0 \text {. }
$$

Since, using Definition 1 for $\underline{\lambda}\left(\sigma ; t^{*}(\lambda \sigma)\right)$ we have $t^{*}(\tilde{\lambda} \sigma)>t^{*}(\lambda \sigma)$ for all $\tilde{\lambda} \in$ $\left[0, \underline{\lambda}\left(\sigma ; t^{*}(\lambda \sigma)\right)\right)$. Then $\underline{\lambda}\left(\sigma ; t^{*}(\lambda \sigma)\right) \leq \lambda$ since otherwise $t^{*}(\lambda \sigma)>t^{*}(\lambda \sigma)$ which is absurd.

The discussion above shows that in addition to the non-sensitivity to non-monotonous behaviour of the durability diagram, the TNESF does not also distinguish rupture from 
not absolutely stable strength. For this reason it is not the durability $t^{*}(\sigma)$ but the critical time $t_{s t}^{*}(\sigma) \leq t^{*}(\sigma)$ which can be obtained from $\underline{\Lambda}(\sigma ; t)$ generally. The following statement is proved in Appendix E.

Statement 3 Let $\sigma_{i j}(\tau)$ be an MD process. The critical time $t_{s t}^{*}(\sigma)$ equals the supremum of $t$ such that

$$
\underline{\Lambda}(\sigma ; t)<1
$$

Taking into account the homogeneity of $\underline{\Lambda}(\sigma ; t)$, one can obtain from Statement 3 the following slightly more general proposition.

Corollary 1 Let $\sigma_{i j}(\tau)$ be an MD process. For any $\lambda>0$, the critical time $t_{s t}^{*}(\lambda \sigma)$ equals the supremum of $t$ such that $\underline{\Lambda}(\sigma ; t)<1 / \lambda$.

The following corollary is proved in Appendix F.

Corollary 2 A time $t^{* *}$ is critical, i.e. $t^{* *}=t_{s t}^{*}(\sigma)$, for an $M D$ process $\sigma_{i j}(\tau)$ if and only if

$$
\underline{\Lambda}(\sigma ; t)<1 \leq \underline{\Lambda}\left(\sigma ; t^{* *}\right) \forall t<t^{* *}
$$

From inequality (15) we also have the following Corollary.

Corollary 3 If $\underline{\Lambda}(\sigma ; t)$ is left-continuous in time at $t=t_{s t}^{*}(\sigma)$ for an $M D$ process $\sigma_{i j}(\tau)$, then $\underline{\Lambda}\left(\sigma ; t_{s t}^{*}(\sigma)\right)=1$.

As was remarked before, one can replace the durability $t^{*}(\lambda \sigma)$ by the critical time $t_{s t}^{*}(\lambda \sigma)$ in Definitions 1,2 to arrive at exactly the same functionals $\underline{\lambda}$ and $\underline{\Lambda}$ for an MD process $\sigma(\tau)$. Thus, if the critical time $t_{s t}^{*}(\lambda \sigma)$ is known for an MD process $\sigma_{i j}(\tau)$ at all $\lambda \geq 0$, then values of the TNESF $\underline{\Lambda}(\sigma ; t)$ are uniquely determined for the process $\sigma_{i j}(\tau)$ at any $t$. Conversely, if values of the TNESF $\underline{\Lambda}(\sigma ; t)$ are known for an MD process $\sigma_{i j}(\tau)$ at all $t$, then values of the critical time $t_{s t}^{*}(\lambda \sigma)$ are uniquely determined for the process $\sigma_{i j}(\tau)$ at any $\lambda \geq 0$ and particularly at $\lambda=1$.

Note that namely the critical time $t_{s t}^{*}(\sigma)$ is necessary for practical design since, as mentioned above, for the cases when $t_{s t}^{*}(\sigma) \neq t^{*}(\sigma)$, the material strength is $\lambda$-unstable for $t \in\left(t_{s t}^{*}(\sigma), t^{*}(\sigma)\right)$.

\section{Existence and uniqueness of the TNESFs.}

Suppose the material strength under a process $\sigma_{i j}(\tau)$ at an instant $t$ is described by a (necessary and sufficient) strength condition

$$
\underline{F}(\sigma ; t)<1
$$


where $\underline{F}$ is a non-linear functional non-decreasing in time, known from experimental data approximation or from a durability theory on the processes $\lambda \sigma_{i j}(\tau)$ for all $\lambda \geq$ 0 and for all instants $t^{\prime \prime}<t^{\prime}$ for some $t^{\prime}>t$. Non-decreasing in time for $F$ means absence of a repairing mechanism. Then necessity and sufficiency of strength condition (16) implies unambiguous detection of the rupture/strength state at any instant $t<t^{\prime}$ and consequently of the durability $t^{*}(\lambda \sigma)$ for all $\lambda \geq 0$ if $t^{*}(\lambda \sigma)<t^{\prime}$. Thus Definitions 1 and 2 are applicable to uniquely determine $\operatorname{TNESF} \underline{\Lambda}(\sigma ; t)$ on $\sigma_{i j}(\tau)$ at that instant $t$, although this not always leads to an analytical expression. Owing to the TNESF homogeneity, we have then its values $\underline{\Lambda}(\lambda \sigma ; t)=\lambda \underline{\Lambda}(\sigma ; t)$ for any $\lambda \geq 0$.

Statement 4 (i) If $\underline{F}(\sigma ; t)$ is a non-negative positively homogeneous functional of order +1 on $\sigma$, then generally $\underline{\Lambda}(\sigma ; t) \geq \underline{F}(\sigma ; t)$.

(ii) If, in addition $\underline{F}(\sigma ; t)$ is right-continuous in the second argument at the considered time $t$, then simply $\underline{\Lambda}(\sigma ; t)=\underline{F}(\sigma ; t)$.

The proof is given in Appendix G.

The statement will be used in Section 6 to obtain TNESFs from known strength conditions of some durability theories.

\section{$6 \quad$ Examples of normalized equivalent stress functionals}

Let us consider examples of calculation of the TNESFs $\underline{\Lambda}$ for several known durability theories. It is supposed for all the examples that $\sigma_{i j}(\tau)=0$ if $\tau \leq 0$.

\subsection{TNESF for constant loading}

\section{Uniaxial constant loading}

For a uniaxial constant loading $\sigma(\tau)=\sigma=$ const. at $\tau>0$, the temporal strength condition can be written in the form

$$
|\sigma|<\sigma^{*}(\operatorname{sign}(\sigma) ; t)
$$

where the temporal strength $\sigma^{*}(\operatorname{sign}(\sigma) ; t)$ is a non-increasing function of time $t$ depending also on the sign of the applied stress $\sigma$. Inversely, the durability condition can be written in the form

$$
t<t^{* 0}(\sigma)
$$

where $|\sigma| \mapsto t^{* 0}(\sigma)=t^{* 0}(\operatorname{sign}(\sigma)|\sigma|)$ is the classical durability diagram for constant loading. 
From (17) and Definition 1, we have the strength condition in terms of TNESF,

$$
\underline{\Lambda}_{0}(\sigma, t)=\frac{|\sigma|}{\sigma^{*}(\operatorname{sign}(\sigma) ; t)}<1
$$

\section{Multiaxial constant loading}

For a multiaxial constant loading $\sigma_{i j}(\tau)=\sigma_{i j}=$ const., $\tau>0$, the temporal strength condition can be written in the form

$$
|\sigma|<\sigma^{*}(\tilde{\sigma} ; t)
$$

where $|\sigma|$ is a matrix norm of the tensor $\sigma_{i j}$, and the temporal strength $\sigma^{*}(\tilde{\sigma} ; t)$ is a non-increasing function of time $t$ depending also on the shape $\tilde{\sigma}_{i j}=\sigma_{i j} /|\sigma|$ of the applied stress tensor $\sigma_{i j}$. Inversely, the durability condition can be written in the form

$$
t<t^{* 0}(\sigma)
$$

where $|\sigma| \mapsto t^{* 0}(\sigma)=t^{* 0}\left(\tilde{\sigma_{i j}}|\sigma|\right)$ is the classical durability diagram for constant multiaxial loading.

From (19) and Definition 1, we have the multiaxial strength condition in terms of TNESF,

$$
\underline{\Lambda}_{0}(\sigma, t)=\frac{|\sigma|}{\sigma^{*}(\tilde{\sigma} ; t)}<1
$$

\subsection{Time and history independent material}

Let the material strength under an arbitrary (right-continuous) multiaxial loading process $\sigma_{i j}(\tau)$ be determined only by its instant stress tensor value. Then it can be described by the strength condition

$$
\Lambda^{I}(\sigma(\tau))<1
$$

where $\Lambda^{I}(\sigma)$ is a known non-negative positively homogeneous function of order +1 . For example, $\Lambda^{I}$ can be the von Mises normalized equivalent stress $\Lambda^{I}(\sigma)=$ $\sqrt{\left[\left(\sigma_{1}-\sigma_{2}\right)^{2}+\left(\sigma_{2}-\sigma_{3}\right)^{2}+\left(\sigma_{3}-\sigma_{1}\right)^{2}\right] /\left(2 \sigma_{r}^{2}\right)}$ or the Tresca normalized equivalent stress $\Lambda^{I}(\sigma)=\max _{k, m}\left|\sigma_{k}-\sigma_{m}\right| / \sigma_{r}$, where, as above, $\sigma_{1}, \sigma_{2}, \sigma_{3}$ are the principal stresses and $\sigma_{r}$ is a known uniaxial tensile strength. Evidently, the strength condition on an interval $0 \leq \tau \leq t$ can be rewritten in the form

$$
\underline{\Lambda}_{1}(\sigma ; t)<1, \quad \underline{\Lambda}_{1}(\sigma ; t)=\sup _{0 \leq \tau \leq t} \Lambda^{I}(\sigma(\tau))
$$

where $\underline{\Lambda}_{1}$ is the TNESF (for instants when it is right-continuous in $t$, cf. Statement 4 ). 


\subsection{Temporal strength condition}

Let the dynamic strength of a material under uniaxial loading be described by the Nikiforovsky-Shemyakin temporal strength condition [10, 11]:

$$
\int_{0}^{t} \sigma(\tau) d \tau<J_{r}
$$

where $J_{r}$ is a material parameter. Then the durability $t^{*}(\lambda \sigma)$ under the process $\lambda \sigma(\tau)$ for $\sigma(\tau)>0$ is determined from the equation

$$
\int_{0}^{t^{*}} \lambda \sigma(\tau) d \tau=J_{r}
$$

and

$$
\lambda=J_{r}\left[\int_{0}^{t^{*}} \sigma(\tau) d \tau\right]^{-1} .
$$

If $\sigma(\tau)>0$ at $\tau>0$, the right hand side of (24) is a continuous monotonously decreasing function of $t^{*}$, and we have from the Definitions $1 \mathrm{CM}$ and 2 ,

$$
\left.\underline{\Lambda}_{2} \sigma ; t\right)=\frac{1}{J_{r}} \int_{0}^{t} \sigma(\tau) d \tau
$$

For arbitrary processes with not necessarily positive $\sigma(\tau)$ at $\tau>0$, we have from the Definitions 1 and 2 a more general formula for the TNESF:

$$
\underline{\Lambda}_{2}(\sigma ; t)=\max \left[0, \frac{1}{J_{r}} \sup _{0 \leq t^{\prime} \leq t} \int_{0}^{t^{\prime}} \sigma(\tau) d \tau\right] .
$$

\subsection{Finitely-temporal strength condition}

Let the dynamic strength of a material under uniaxial loading be described by the finitelytemporal (structural-temporal) strength condition (see [12]) at an instant $t$ :

$$
\sup _{0 \leq t^{\prime} \leq t} \frac{1}{t_{r}} \int_{t^{\prime}-t_{r}}^{t^{\prime}} \sigma(\tau) d \tau<\sigma_{r}
$$

where $t_{r}>0$ and $\sigma_{r}>0$ are material parameters. Then the durability $t^{*}(\lambda \sigma)$ under the process $\lambda \sigma(\tau)$ is determined as a minimal non-negative solution of the equation

$$
\sup _{0 \leq t^{\prime} \leq t^{*}} \frac{1}{t_{r}} \int_{t^{\prime}-t_{r}}^{t^{\prime}} \lambda \sigma(\tau) d \tau=\sigma_{r}
$$

and

$$
\lambda=\sigma_{r} t_{r}\left[\sup _{0 \leq t^{\prime} \leq t^{*}} \int_{t^{\prime}-t_{r}}^{t^{\prime}} \sigma(\tau) d \tau\right]^{-1} .
$$

From the Definitions 1 and 2, then the TNESF is

$$
\underline{\Lambda}_{3}(\sigma ; t)=\max \left[0, \frac{1}{\sigma_{r} t_{r}} \sup _{0 \leq t^{\prime} \leq t} \int_{t^{\prime}-t_{r}}^{t^{\prime}} \sigma(\tau) d \tau\right] .
$$

It is evident that $\underline{\Lambda}_{2}(\sigma ; t)=\underline{\Lambda}_{3}(\sigma ; t)$ for $t \leq t_{r}$ if $J_{r}=\sigma_{r} t_{r}$. 


\subsection{Campbell strength condition}

Campbell [13] introduced the following yielding criterion under uniaxial dynamic loading $\sigma(\tau) \geq 0$

$$
\int_{0}^{t^{*}}\left[\frac{\sigma(\tau)}{\sigma_{r}}\right]^{b} d \tau=t_{r}
$$

where $t_{r}>0, \sigma_{r}>0$ and $b$ are material parameters. We can treat this also as a dynamic rupture criterion. Then the corresponding strength condition is

$$
\underline{\Lambda}_{4}(\sigma ; t)<1
$$

where

$$
\underline{\Lambda}_{4}(\sigma ; t)=\left[\frac{1}{t_{r}} \int_{0}^{t}\left[\frac{\sigma(\tau)}{\sigma_{r}}\right]^{b} d \tau\right]^{1 / b}
$$

is the TNESF, Statement 4.

If $b=1$, then evidently, $\underline{\Lambda}_{4}(\sigma ; t)$ degenerates into $\underline{\Lambda}_{2}(\sigma ; t)$ for $J_{r}=\sigma_{r} t_{r}$. For $b=1$ and $t \leq t_{r}$ it coincides also with $\underline{\Lambda}_{3}(\sigma ; t)$.

\subsection{Modified Campbell strength condition}

A finitely temporal modification of dynamic yield (rapture) criterion (30) presented in [12] (see also references therein),

$$
\frac{1}{t_{r}} \int_{t^{*}-t_{r}}^{t^{*}}\left[\frac{\sigma(\tau)}{\sigma_{r}}\right]^{b} d \tau=1
$$

leads to the following strength condition,

$$
\underline{\Lambda}_{5}(\sigma ; t)<1
$$

where

$$
\underline{\Lambda}_{5}(\sigma ; t)=\sup _{0 \leq t^{\prime} \leq t}\left[\frac{1}{t_{r}} \int_{t^{\prime}-t_{r}}^{t^{\prime}}\left[\frac{\sigma(\tau)}{\sigma_{r}}\right]^{b} d \tau\right]^{1 / b}
$$

is the TNESF and $t_{r}>0, \sigma_{r}>0$ and $b$ are material parameters. If $b=1$, then evidently, $\underline{\Lambda}_{5}(\sigma ; t)$ degenerates into $\underline{\Lambda}_{3}(\sigma ; t)$. For $t \leq t_{r}$ it coincides also with $\underline{\Lambda}_{4}(\sigma ; t)$ and if, additionally, $b=1$, then also with $\underline{\Lambda}_{2}(\sigma ; t)$ for $J_{r}=\sigma_{r} t_{r}$.

\subsection{Il'ushin durability theory}

\subsubsection{Linear theory}

It was supposed in [5] that there exists an abstract damage tensor $\omega_{i j}(\sigma ; t)$ which is a functional defined on load processes $\sigma_{i j}(\tau)$ (for simplicity, we neglect here dependence of 
$\omega$ also on stress moments considered in [5]). It was supposed that there is no rupture if a set of $\tilde{m}$ strength conditions written in terms of $\omega(\sigma ; t)$ is satisfied:

$$
M_{m}(\omega)<c_{m}, \quad m=1, \ldots, \tilde{m} .
$$

Here functions $M_{m}$ and constants $c_{m}$ are material characteristics associated with an $m$-th rupture mode.

It was supposed in the linear version of the Il'ushin theory [5] that the tensor damage functional $\omega(\sigma ; t)$ can be taken in the form

$$
\omega_{i j}(\sigma ; t)=\int_{0}^{t} \varphi_{i j k l}(t-\tau) d \sigma_{k l}(\tau)
$$

where functions $\varphi_{i j k l}(\tau)$ are material characteristics independent of $\sigma_{i j}(\tau)$.

Denoting $M\left(\omega_{i j}\right)=\max _{m=1, \ldots \tilde{m}}\left(M_{m}\left(\omega_{i j}\right) / c_{m}\right)$, we can rewrite $(36)$ - (37) in the form

$$
M\left[\int_{0}^{t} \varphi_{i j k l}(t-\tau) d \sigma_{k l}(\tau)\right]<1 .
$$

Since the left hand side of (38) can be non-monotonous in $t$ at least for non-monotonous processes $\sigma_{k l}(\tau)$, it should be corrected so as not to predict a life after rupture, e.g., in the following way,

$$
\sup _{0 \leq t^{\prime} \leq t} M\left[\int_{0}^{t^{\prime}} \varphi_{i j k l}\left(t^{\prime}-\tau\right) d \sigma_{k l}(\tau)\right]<1 .
$$

The corresponding strength condition for a process $\lambda \sigma_{i j}(\tau)$ takes the form

$$
\sup _{0 \leq t^{\prime} \leq t} M\left[\lambda \int_{0}^{t^{\prime}} \varphi_{i j k l}\left(t^{\prime}-\tau\right) d \sigma_{k l}(\tau)\right]<1 .
$$

Suppose first that the function $M$ is non-negative and positively homogeneous of the order +1 , i.e., $M\left(\lambda \omega_{i j}\right)=\lambda M\left(\omega_{i j}\right)$. Then according to Definitions $1 \mathrm{CM}$ and 2 , we have the following expression for the TNESF:

$$
\underline{\Lambda}_{6}(\sigma ; t)=\sup _{0 \leq t^{\prime} \leq t} M\left[\int_{0}^{t^{\prime}} \varphi_{i j k l}\left(t^{\prime}-\tau\right) d \sigma_{k l}(\tau)\right] .
$$

If the function $M$ is not positively homogeneous of the order +1 , one should reduce (38) to an equivalent form with a new function $M$ which is already homogeneous. Another way is to apply more general Definition 1 , what probably demands some numerical calculations. In this case $\underline{\lambda}_{6}(\sigma ; t)$ is supremum of numbers $\lambda^{*}$ such that inequality (40) is satisfied for all $\lambda \in\left[0, \lambda^{*}\right]$. If $M\left(\lambda \omega_{i j}\right)$ is continuously monotonously growing with $\lambda$, then one can get $\underline{\lambda}_{6}(\sigma ; t)$ more simply as a solution of the equation obtained from (40) (after replacing the sign " $<$ " by the sign " ="), instead of finding the supremum. Then $\underline{\Lambda}_{6}(\sigma ; t)=1 / \underline{\lambda}_{6}(\sigma ; t)$ according to Definition 2.

Suppose $\sigma_{11}(\tau)$ is a uniaxial process. If we take $\varphi_{i j k l}(t-\tau)=(t-\tau) \delta_{i k} \delta_{j l}$ and $M\left(\omega_{11}\right)=$ $\omega_{11} / J_{r}$, then the TNESF $\underline{\Lambda}_{6}(\sigma ; t)$ from the Il'ushin linear durability theory coincides with 
its counterpart $\underline{\Lambda}_{2}(\sigma ; t)$ given by the Nikiforovsky-Shemyakin temporal strength condition. On the other hand, if we take $\varphi_{i j k l}(t-\tau)=\left[t-\tau-\left(t-\tau-t_{r}\right) H\left(t-\tau-t_{r}\right)\right] \delta_{i k} \delta_{j l}$ and $M\left(\omega_{11}\right)=\omega_{11} /\left(\sigma_{r} t_{r}\right)$, then the TNESF $\underline{\Lambda}_{6}(\sigma ; t)$ from the Il'ushin linear durability theory coincides with its counterpart $\underline{\Lambda}_{3}(\sigma ; t)$ given by the finitely-temporal strength condition (27).

\subsubsection{Non-linear theory}

In the non-linear version of the Il'ushin durability theory [5], representation (37) is replaced by a more general non-linear form for the damage tensor functional:

$$
\omega_{i j}(\sigma ; t)=\sum_{n=1}^{\infty} \int_{0}^{t} \ldots \int_{0}^{t} \Phi_{i j i_{1} j_{1} \ldots i_{n} j_{n}}^{(n)}\left(t-\tau_{1}, \ldots, t-\tau_{n}\right) \sigma_{i_{1} j_{1}}\left(\tau_{1}\right) \ldots \sigma_{i_{n} j_{n}}\left(\tau_{n}\right) d \tau_{1} \ldots d \tau_{n}
$$

Then as above, the corresponding strength condition for a process $\lambda \sigma_{i j}(\tau)$ takes the form

$$
\sup _{0 \leq t^{\prime} \leq t} M\left[\sum_{n=1}^{\infty} \lambda^{n} \int_{0}^{t^{\prime}} \ldots \int_{0}^{t^{\prime}} \Phi_{i j i_{1} j_{1} \ldots i_{n} j_{n}}^{(n)}\left(t^{\prime}-\tau_{1}, \ldots, t^{\prime}-\tau_{n}\right) \sigma_{i_{1} j_{1}}\left(\tau_{1}\right) \ldots \sigma_{i_{n} j_{n}}\left(\tau_{n}\right) d \tau_{1} \ldots d \tau_{n}\right]<1 .
$$

If the left hand side of (43) is a monotonously and continuously growing function of $\lambda$, then, according to Definition $1 \mathrm{CM}$, one can get $\underline{\lambda}_{6}(\sigma ; t)$ as a solution of the equation obtained from (43) (after replacement the sign " <" by the sign " =" there). Otherwise, one can apply more general Definition 1 . In this case $\underline{\lambda}_{6}(\sigma ; t)$ is the supremum of the numbers $\lambda^{*}$ such that inequality (43) is satisfied for all $\lambda \in\left[0, \lambda^{*}\right]$. Then $\underline{\Lambda}_{6}(\sigma ; t)=1 / \underline{\lambda}_{6}(\sigma ; t)$ according to Definition 2.

\subsection{Robinson linear rule of damage accumulation}

Let a material obey the Robinson hypothesis of creep damage linear accumulation $[14,15]$ (see also $[3,16])$. Then the durability $t^{*}(\sigma)$ under a multiaxial process $\sigma_{i j}(\tau)$ can be determined from the equation

$$
\int_{0}^{t^{*}} \frac{d \tau}{t^{* 0}(\sigma(\tau))}=1
$$

Here $t^{* 0}(\sigma(\tau))=t^{* 0}\left(\sigma_{i j}(\tau)\right)=\left.t^{*}\left(\sigma_{i j}^{0}\right)\right|_{\sigma_{i j}^{0}=\sigma_{i j}(\tau)}$ is a function presenting the classical durability diagram under a multiaxial step-like loading, where $\sigma_{i j}^{0}=$ const. Then $t^{*}(\lambda \sigma)$ is determined from equation

$$
\int_{0}^{t^{*}} \frac{d \tau}{t^{* 0}(\lambda \sigma(\tau))}=1
$$

Suppose the classical durability diagram $t^{*}\left(\sigma_{i j}^{0}\right)$ is given by the power law $(1)$. Then (45) is reduced to

$$
\int_{0}^{t^{*}} \frac{(\lambda|\sigma(\tau)|)^{b(\tilde{\sigma}(\tau))}}{A(\tilde{\sigma}(\tau))} d \tau=1
$$


Suppose additionally that $\sigma_{i j}(\tau)$ is an in-phase (coaxial, proportional) multiaxial process, that is, the shape of the tensor $\sigma_{i j}(\tau)$ does not vary in time: $\sigma_{i j}(\tau)=\sigma_{i j}^{0} \frac{|\sigma(\tau)|}{\left|\sigma^{0}\right|}$, that is, $\tilde{\sigma}_{i j}(\tau)=\sigma_{i j}(\tau) /|\sigma(\tau)|=\sigma_{i j}^{0} /\left|\sigma^{0}\right|=$ const. Then $A(\tilde{\sigma}(\tau))=$ const, $b(\tilde{\sigma}(\tau))=$ const and we have from (46),

$$
\lambda=\left[\frac{1}{A(\tilde{\sigma})} \int_{0}^{t^{*}}|\sigma(\tau)|^{b(\tilde{\sigma})} d \tau\right]^{-1 / b(\tilde{\sigma})} .
$$

Suppose $b>0$. Then the right hand side of (47) is a continuous monotonously nonincreasing function of $t^{*}$. From the Definitions 1CM and 2, we then have the following representations for the TNESF on in-phase processes,

$$
\underline{\Lambda}_{7}(\sigma ; t)=\left[\frac{1}{A(\tilde{\sigma})} \int_{0}^{t}|\sigma(\tau)|^{b(\tilde{\sigma})} d \tau\right]^{1 / b(\tilde{\sigma})} .
$$

Let now $\sigma_{i j}(\tau)$ be an arbitrary multiaxial process but $b=$ const is a positive material parameter independent on $\tilde{\sigma}_{i j}(\tau)$. Then in the similar way one obtains for this case the TNESF

$$
\underline{\Lambda}_{7}(\sigma ; t)=\left[\int_{0}^{t} \frac{|\sigma(\tau)|^{b}}{A(\tilde{\sigma}(\tau))} d \tau\right]^{1 / b} .
$$

If $\sigma(\tau) \geq 0$ is a uniaxial process, then TNESFs (48), (49) coincide for $A=t_{r} \sigma_{r}^{b}$ with the functional $\underline{\Lambda}_{4}(\sigma ; t)$ associated with the Campbell strength condition.

If the loading is not in-phase and $b(\tilde{\sigma})$ is not constant or the classical durability diagram is more complicated than (1), then equation (45) can not be generally solved with respect to $\lambda$ analytically but this can be done numerically. The solution gives $\underline{\lambda}_{7}\left(\sigma ; t^{*}\right)$ and $\underline{\Lambda}_{7}\left(\sigma ; t^{*}\right)=1 / \underline{\lambda}_{7}\left(\sigma ; t^{*}\right)$, if $t^{* 0}\left(\lambda \sigma_{i j}(\tau)\right)$ is a decreasing function of $\lambda$. Otherwise one should apply general Definitions 1 and 2 to (45) (where the sign "=" must be replaced by the sign "<").

\subsection{Hoff model for rod creep rupture}

Consider an incompressible rod under a nominal stress $\sigma_{0}(\tau) \geq 0$ at $\tau>0$. Its creep can be described by the Norton creep law

$$
\frac{d \epsilon(\tau)}{d \tau}=a \sigma^{b}(\tau)
$$

$\epsilon$ is the creep logarithmic strain, $a, b>0$ are material constants. The creep rupture is modelled by Hoff [17] (see also [3, Section 85], [4, Section 2.2]) taking into account the increase of the actual stress $\sigma$, caused by the rod cross-section decrease due to the material incompressibility,

$$
\sigma=\sigma_{0}(\tau) e^{\epsilon(\tau)}
$$

The strength condition

$$
\sup _{0 \leq \tau \leq t} \sigma(\tau)<\infty
$$


is applied, which can be also rewritten in the form

$$
\underline{\Lambda}_{8}(\sigma ; t)<1, \quad \underline{\Lambda}_{8}(\sigma ; t)=\left\{\begin{array}{lll}
0 & \text { if } & \sup _{0 \leq \tau \leq t} \sigma(\tau)<\infty \\
\infty & \text { if } \sup _{0 \leq \tau \leq t} \sigma(\tau)=\infty
\end{array}\right.
$$

Substitution (51) into (50) and its integration with the initial condition $\epsilon(0)=0$ gives the following relation between actual and nominal stresses

$$
\sigma(t)=\sigma_{0}(t)\left[1-a b \int_{0}^{t} \sigma_{0}^{b}(\tau) d \tau\right]^{-1 / b} .
$$

Using (52), we arrive for a process $\lambda \sigma_{0}(\tau)$ at the strength condition in terms of $\sigma_{0}$ on a time segment $[0, t]$ :

$$
\sup _{0 \leq \tau \leq t} \lambda \sigma_{0}(\tau)\left[1-a b \lambda^{b} \int_{0}^{\tau} \sigma_{0}^{b}(\xi) d \xi\right]^{-1 / b}<\infty .
$$

This means that, in terms of the nominal stress, the TNESF for this model is

$$
\underline{\Lambda}_{08}\left(\sigma_{0} ; t\right)=\max \left\{\underline{\hat{\Lambda}}_{08}\left(\sigma_{0} ; t\right), \underline{\Lambda}_{8}\left(\sigma_{0} ; t\right)\right\}
$$

where

$$
\underline{\hat{\Lambda}}_{08}\left(\sigma_{0} ; t\right)=\left[a b \int_{0}^{t} \sigma_{0}^{b}(\tau) d \tau\right]^{1 / b} .
$$

Up to the notation $a b=1 / A=1 /\left(t_{r} \sigma_{r}\right)$, the functional $\underline{\hat{\Lambda}}_{08}$ coincides with the TNESF $\underline{\Lambda}_{4}(\sigma ; t)$ corresponding to the Campbell strength condition and with the functional $\underline{\Lambda}_{7}(\sigma ; t)$ corresponding to the power law of durability and the linear rule of damage accumulation.

\subsection{Kachanov damage model}

In the Kachanov damage model [1] (see also, [3, Section 87], [4, Section 2.4]) the same problem as in the Hoff model is considered and the same creep low (50) and expression for the actual stress (51) are supposed. However the strength condition (52) is replaced by the strength condition

$$
\sup _{0 \leq \tau \leq t} \frac{\sigma(\tau)}{1-\omega(\tau)}<\infty
$$

which is equivalent to (52) supplemented by the strength condition

$$
\omega(t)<1
$$

Here $\omega(t)$ is a damage measure, which behaviour is described by equation

$$
\frac{d \omega(\tau)}{d \tau}=B\left(\frac{\sigma(\tau)}{1-\omega(\tau)}\right)^{k}
$$

with the initial condition $\omega(0)=0 ; B, k>0$ are material constants. 
Integrating (60), we can after some manipulations rewrite the strength condition (59) in the form homogeneous with respect to $\sigma$,

$$
\underline{\hat{\Lambda}}_{9}(\sigma ; t)<1, \quad \underline{\hat{\Lambda}}_{9}(\sigma ; t)=\left[(k+1) B \int_{0}^{t} \sigma^{k}(\tau) d \tau\right]^{1 / k} .
$$

Note that $\underline{\hat{\Lambda}}_{9}$ coincides with $\underline{\Lambda}_{4}, \underline{\Lambda}_{7}$ and $\underline{\hat{\Lambda}}_{08}$ up to notations.

Recalling the strength condition (53), we finally obtain the TNESF in terms of the actual stress $\sigma$,

$$
\underline{\Lambda}_{9}(\sigma ; t)=\max \left(\underline{\Lambda}_{8}(\sigma ; t), \underline{\hat{\Lambda}}_{9}(\sigma ; t)\right) .
$$

To obtain the TNESF in terms of the nominal stress, we substitute (54) in (61) and arrive for a process $\lambda \sigma_{0}(\tau)$ at the strength condition

$$
\lambda^{k} \int_{0}^{t} \sigma_{0}^{k}(\tau)\left[1-a b \lambda^{b} \int_{0}^{\tau} \sigma_{0}^{b}(\xi) d \xi\right]^{-k / b} d \tau<\frac{1}{(k+1) B}
$$

completing strength condition (55) on a time segment $[0, t]$.

Taking into account (55) and condition $\sigma_{0} \geq 0$, one can see that the left hand side of (63) is a monotonously increasing function of $\lambda$ at fixed $t$ and is a monotonously nondecreasing function $t$ at fixed $\lambda$. This means the TNESF in terms of the nominal stress for this model is

$$
\underline{\Lambda}_{09}\left(\sigma_{0} ; t\right)=\max \left(\underline{\Lambda}_{08}\left(\sigma_{0} ; t\right), 1 / \lambda_{09}\left(\sigma_{0} ; t\right)\right) .
$$

where $\underline{\Lambda}_{08}\left(\sigma_{0} ; t\right)$ is defined in $(56)$ and $\lambda_{09}\left(\sigma_{0} ; t\right)$ is a unique non-negative solution of the equation obtained from (63) after replacement of the inequality by the equality sign (for instants when it is right-continuous in $t$, see Statement 4). For each $t$ and an arbitrary process $\sigma_{0}(\tau) \geq 0$, this equation is nonlinear transcendental and can be solved numerically. For $\sigma_{0}(\tau)=$ const at $\tau \geq 0$, this equation is reduced to

$$
t=\frac{1}{a b\left(\lambda \sigma_{0}\right)^{b}}\left\{1-\left[1-\frac{(b-k) a\left(\lambda \sigma_{0}\right)^{b-k}}{(k+1) B}\right]^{\frac{b}{b-k}}\right\},
$$

cf. [1], [3, Section 87], [4, Section 2.4].

\subsection{Rabotnov damage model}

In the Rabotnov damage model [2] (see also [3, Section 87] and [4, Section 2.4]), the same problem for a rod under creep, as in the Hoff and Kachanov models is considered. However an influence of the damage on the creep is taken into account in the form

$$
\frac{d \epsilon(\tau)}{d \tau}=a \sigma^{b}(\tau)(1-\omega(\tau))^{-q}
$$

whereas the relation between the nominal $\sigma_{0}$ and actual $\sigma$ stresses is given by the same formula (51). 
An expression for the damage rate more general than (60) is given in the form

$$
\frac{d \omega(\tau)}{d \tau}=B \sigma^{k}(\tau)(1-\omega(\tau))^{-r}
$$

Here $a, b, q, B, k, r$ are considered as material constants. It is evident that (65), (66) degenerate at $q=0, k=r$ into the relations (50), (60) used by Kachanov.

The strength condition (59) completed in fact by condition (52) was used by Rabotnov on a time segment $[0, t]$.

Integrating (66), we get

$$
[1-\omega(t)]^{r+1}=1-(r+1) B \int_{0}^{t} \sigma^{k}(\tau) d \tau
$$

Then we can rewrite the strength condition (59) in the form

$$
\underline{\Lambda}_{10}(\sigma ; t)<1, \quad \underline{\hat{\Lambda}}_{10}(\sigma ; t)=\left[(r+1) B \int_{0}^{t} \sigma^{k}(\tau) d \tau\right]^{1 / k}
$$

Recalling the strength condition (53) equivalent to (52), we finally obtain the TNESF for the Rabotnov model in terms of the actual stress $\sigma$,

$$
\underline{\Lambda}_{10}(\sigma ; t)=\max \left(\underline{\Lambda}_{8}(\sigma ; t), \underline{\Lambda}_{10}(\sigma ; t)\right)
$$

To obtain the TNESF in terms of the nominal stress, we first substitute (51) in (65), and integrate. Using the resulting expression for $\epsilon$ in (51) gives,

$$
\sigma(\tau)=\sigma_{0}(\tau)\left\{1-a b \int_{0}^{\tau} \sigma_{0}^{b}(\xi)[1-\omega(\xi)]^{-q} d \xi\right\}^{-1 / b}
$$

Substituting this in (66), after integration we obtain a non-linear integral equation connecting $\omega$ with $\sigma_{0}$,

$$
[1-\omega(t)]^{r+1}=1-(r+1) B \int_{0}^{t} \sigma_{0}^{k}(\tau)\left\{1-a b \int_{0}^{\tau} \sigma_{0}^{b}(\xi)[1-\omega(\xi)]^{-q} d \xi\right\}^{-k / b} d \tau .
$$

Let $\omega^{\prime \prime}$ be a solution of the equation

$$
\left[1-\omega^{\prime \prime}(t)\right]^{r+1}=1-(r+1) B \int_{0}^{t}\left(\lambda^{\prime \prime} \sigma_{0}(\tau)\right)^{k}\left\{1-a b \int_{0}^{\tau}\left(\lambda^{\prime \prime} \sigma_{0}\right)^{b}(\xi)\left[1-\omega^{\prime \prime}(\xi)\right]^{-q} d \xi\right\}^{-k / b} d \tau \text {. }
$$

Strength condition (59) for $\omega^{\prime \prime}$ generated by the process $\lambda^{\prime \prime} \sigma_{0}(\tau)$ gives

$$
\lambda^{\prime \prime k} \int_{0}^{t} \sigma_{0}^{k}(\tau)\left\{1-a b \lambda^{\prime \prime b} \int_{0}^{\tau} \sigma_{0}^{b}(\xi)\left[1-\omega^{\prime \prime}(\xi)\right]^{-q} d \xi\right\}^{-k / b} d \tau<\frac{1}{(r+1) B} .
$$

Inequality (73) is reminiscent of (63) but there is the additional multiplier $\left[1-\omega^{\prime \prime}\right]^{-q}$ in (73), which also depends on $\lambda^{\prime \prime}$ through (72). 
Let us define a functional $\hat{\underline{\lambda}}_{010}\left(\sigma_{0} ; t\right)$, according to the Definition 1 , as the supremum of non-negative numbers $\lambda$ such that inequality (73), where $\omega^{\prime \prime}$ is a solution of (72), is satisfied for any $\lambda^{\prime \prime} \in[0, \lambda]$. The functional $\underline{\hat{\lambda}}_{010}$ can be also equivalently defined as a minimal positive solution $\lambda^{\prime \prime}$ of (72) and the corresponding equality obtained from (73), if the solution does exist.

Owing to (70), strength condition (52) for $\lambda^{\prime \prime} \sigma_{0}$ gives

$$
\sup _{0 \leq \tau \leq t} \sigma_{0}(\tau)\left\{1-a b \lambda^{\prime \prime b} \int_{0}^{\tau} \sigma_{0}^{b}(\xi)\left[1-\omega^{\prime \prime}(\xi)\right]^{-q} d \xi\right\}^{-1 / b}<\infty
$$

where $\omega^{\prime \prime}$ is a solution of $(72)$. Let a functional $\underline{\hat{\lambda}}_{010}^{0}\left(\sigma_{0} ; t\right)$ be the supremum of non-negative numbers $\lambda$ such that inequality

$$
\lambda^{\prime \prime}<\sup _{0 \leq \tau \leq t}\left\{a b \int_{0}^{\tau} \sigma_{0}^{b}(\xi)\left[1-\omega^{\prime \prime}(\xi)\right]^{-q} d \xi\right\}^{1 / b}
$$

where $\omega^{\prime \prime}$ is a solution of $(72)$, is satisfied for any $\lambda^{\prime \prime} \in[0, \lambda]$. The functional $\hat{\lambda}_{010}^{0}$ can be equivalently defined as a minimal positive solution $\lambda^{\prime \prime}$ of (72) and the corresponding equality obtained from (75), if the solution does exist. Finally the TNESF in terms of the nominal stress for the Rabotnov model has the form

$$
\underline{\Lambda}_{010}\left(\sigma_{0} ; t\right)=\max \left(\underline{\Lambda}_{08}\left(\sigma_{0} ; t\right), 1 / \underline{\hat{\lambda}}_{010}^{0}\left(\sigma_{0} ; t\right), 1 / \underline{\hat{\lambda}}_{010}\left(\sigma_{0} ; t\right)\right)
$$

(for instants when it is right-continuous in $t$, see Statement 4).

Examples 6.8-6.10 particularly show that the TNESF and corresponding strength condition for the same material (or model) can look quite different being presented in terms of the nominal or actual stress and one should always carefully fix the used stress type.

\subsection{TNESFs for other damage models}

One of the general forms of the continuum damage mechanics (see e.g. $[3,4,6,7]$ ) can be written as an expression of effective (micro-)stress tensor $\tilde{\sigma}(\tau)$ in terms of the actual (macro-)stress tensor $\sigma(\tau)$ and a damage (tensor) measure $\omega(\tau)$

$$
\tilde{\sigma}(\tau)=f_{1}(\omega, \sigma)
$$

a damage rate equation

$$
\frac{d \omega(\tau)}{d \tau}=f_{2}(\omega, \sigma)
$$

with the initial condition

$$
\omega(0)=0,
$$

and a (necessary and sufficient) strength condition

$$
F(\omega ; \sigma)<1
$$


The functions $f_{1}, f_{2}$, and $F$ are considered to be known material characteristics. The models described in Sections 6.9 and 6.10 present particular cases of (77)-(80). To complete the problem, corresponding constitutive equations of the material and equilibrium equations written in terms of $\sigma$ or $\tilde{\sigma}$, should be added to (77)-(80). However, we need only equations (78), (79), and strength condition (80) to determine the TNESF for such a model in terms of the actual (macro-)stress. Since, integrating (78) with the initial conditions (79) we get, as in subsections 6.9, 6.10,

$$
\omega(t)=f_{3}(\sigma ; t)
$$

where the functional $f_{3}$ is a solution of (78), (79) for a given process $\sigma(\tau)$. Substituting this in (80), we get the following strength condition for a process $\sigma(\tau)$,

$$
F\left(f_{3}(\sigma ; t) ; \sigma\right)<1
$$

Assuming absence of a repairing mechnaism, this is equivalent to the condition

$$
\sup _{0 \leq t^{\prime} \leq t} F\left(f_{3}\left(\sigma ; t^{\prime}\right) ; \sigma\right)<1
$$

Then the left hand side of (82) is non-decreasing in time and the TNESF can be obtained from Definitions 1-2, see Section 5.

Note that although the TNESF is determined in this way independently of the equation (77) and of the material constitutive and equilibrium equations, the equations will be necessary to determine the process $\sigma_{i j}(\tau)$ and to calculate a corresponding value of the TNESF.

\section{Complex TNESF for combined creep, instant and dynamic loading}

The TNESF $\underline{\Lambda}^{T}$ is a material characteristic which is not necessary connected with a geometrical, stiffness-related or abstract damage measure and can be identified from some durability tests. As shown in the previous sections, any strength condition written in terms of a damage measure can be expressed in terms of a corresponding TNESF (although not always analytically). Let us show some simple ways constructing TNESFs to include e.g. instant overloading or dynamic effects in addition to creep durability. The Robinson damage accumulation rules mentioned above did not take into account sequence effects, that is damage caused by a stress in a particular instant is independent of where it occurs in the load history. We will see that this shortcoming can be overcome in a simple way by choosing a proper structure of TNESFs. 
Suppose one has a TNESF $\underline{\Lambda}^{T}(\sigma ; t)$ obtained e.g. from a damage measure approach, which do not take into account any influence of instantaneous overloads of material, especially a finite strength under instantaneous loading. Particularly, TNESFs (48) and (49) based on the power-type durability diagrams give such examples. To avoid this shortcoming, one can combine an instant normalized equivalent stress function $\Lambda^{I}$ and a temporal TNESF $\underline{\Lambda}^{T}$ and arrive at a complex strength condition e.g. in the form

$$
\underline{\Lambda}^{I T}(\sigma ; t)=\sup _{0 \leq t^{\prime} \leq t}\left\{\Lambda^{I}\left(\sigma\left(t^{\prime}\right)\right)+\underline{\Lambda}^{T}\left(\sigma ; t^{\prime}\right)\right\}<1 .
$$

For example, if $\Lambda^{I}(\sigma)=\sigma_{e q}(\sigma) / \sigma_{r}, \sigma_{e q}(\sigma)$ is von Mises, Tresca or other instantaneous equivalent stress, $\sigma_{r}$ is an instant uniaxial strength, and $\underline{\Lambda}^{T}(\sigma ; t)$ is given by (49), then the TNESF (83) will take form

$$
\underline{\Lambda}^{I T}(\sigma ; t)=\sup _{0 \leq t^{\prime} \leq t}\left\{\frac{\sigma_{e q}\left(\sigma\left(t^{\prime}\right)\right)}{\sigma_{r}}+\left[\int_{0}^{t^{\prime}} \frac{|\sigma(\tau)|^{b}}{A(\tilde{\sigma}(\tau))} d \tau\right]^{1 / b}\right\} .
$$

where $b$ is a material parameter and $A(\tilde{\sigma}(\tau))$ is a material functions of the normalized stress tensor $\tilde{\sigma}_{i j}(\tau)=\sigma_{i j}(\tau) /|\sigma(\tau)|$ at an instant $\tau$.

If there exist also dynamic effects on the material strength and the instant strength is not well defined, one can replace the instant strength term by a corresponding dynamic TNESF $\underline{\Lambda}^{D}\left(\sigma\left(t^{\prime}\right)\right.$ and arrive e.g. at the following complex TNESF and strength condition,

$$
\underline{\Lambda}^{D T}(\sigma ; t)=\sup _{0 \leq t^{\prime} \leq t}\left\{\underline{\Lambda}^{D}\left(\sigma ; t^{\prime}\right)+\underline{\Lambda}^{T}\left(\sigma ; t^{\prime}\right)\right\}<1
$$

For example, we can take $\underline{\Lambda}^{T}\left(\sigma ; t^{\prime}\right)$ in form (49), and $\underline{\Lambda}^{D}\left(\sigma ; t^{\prime}\right)$ associated with the Morozov, Petrov and Utkin dynamic strength condition (27) generalised on the multiaxial case in the form

$$
\underline{\Lambda}^{D}\left(\sigma ; t^{\prime}\right)=\frac{1}{\sigma_{r}} \sigma_{e q}\left(\bar{\sigma}\left(t^{\prime} ; t_{r}\right)\right), \quad \bar{\sigma}_{k j}\left(t^{\prime} ; t_{r}\right)=\frac{1}{t_{r}} \int_{t^{\prime}-t_{r}}^{t^{\prime}} \sigma_{k j}\left(t^{\prime \prime}\right) d t^{\prime \prime},
$$

where $\sigma_{e q}(\sigma)$ is e.g. von Mises, Tresca or other instantaneous equivalent stress and $\sigma_{r}, t_{r}$ are a material constants. Then the TNESF (85) will take form

$$
\underline{\Lambda}^{D T}(\sigma ; t)=\sup _{0 \leq t^{\prime} \leq t}\left\{\frac{1}{\sigma_{r}} \sigma_{e q}\left(\bar{\sigma}\left(t^{\prime} ; t_{r}\right)\right)+\left[\int_{0}^{t^{\prime}} \frac{|\sigma(\tau)|^{b}}{A(\tilde{\sigma}(\tau))} d \tau\right]^{1 / b}\right\} .
$$

Note that both strength conditions (84) and (87) lead to non-linear summation rules since they include the fading memory terms $\sigma_{e q}\left(\sigma\left(t^{\prime}\right)\right) / \sigma_{r}$ or $\sigma_{e q}\left(\bar{\sigma}\left(t^{\prime} ; t_{r}\right) / \sigma_{r}\right)$.

Note also that presentations (83) and (85) are not uniquely possible and one can use not only the sum but also other homogeneous combinations of the terms $\Lambda^{I}, \underline{\Lambda}^{D}$ and $\underline{\Lambda}^{T}$ to get other possible simple forms of the TNESFs describing interaction of instant, dynamic and long-time effects on the durability. For example, one can take,

$$
\underline{\Lambda}^{D T}(\sigma ; t)=\sup _{0 \leq t^{\prime} \leq t}\left\{\left[\underline{\Lambda}^{D}\left(\sigma ; t^{\prime}\right)\right]^{q}+\left[\underline{\Lambda}^{T}\left(\sigma ; t^{\prime}\right)\right]^{q}\right\}^{\frac{1}{q}}
$$


instead of (85), where $q>0$ can be considered as a material parameter. If $q=1$, (88) is reduced to (85). The limiting case $q \rightarrow \infty$ corresponds to the TNESF

$$
\underline{\Lambda}^{D T}(\sigma ; t)=\sup _{0 \leq t^{\prime} \leq t} \max \left\{\underline{\Lambda}^{D}\left(\sigma ; t^{\prime}\right), \underline{\Lambda}^{T}\left(\sigma ; t^{\prime}\right)\right\}
$$

Evidently, which form fits better to a particular material behaviour, can be determined from comparison with experimental data.

\section{Conclusion and perspectives}

The generalised durability diagrams introduced in the paper give an instrument to compare irregular loading processes of different intensities and allow the introduction of notions of the temporal safety factor and the temporal normalized equivalent stress for such processes. The TNESF is a mechanically meaningful material characteristic, which can be determined from the durability macro-experiments without any additional information such as micro-cracks or micro-voids distribution or stiffness change. The concept of normalized equivalent stress forms a basis for the durability and strength description under creep and/or dynamic loadings, which do not need the introduction of any damage measures. On the other hand, the durability analysis based on damage measures, is reduced to a particular case of the normalized equivalent stress concept. Nevertheless, the continuum damage mechanics remains helpful also when the normalized equivalent stress concept is applied. Particularly, the softening damage measures allow the calculation of a stress redistribution in a structure element and the geometrical damage measures can be used for estimation of macroscopic TNESFs from micro-mechanical modelling.

The TNESF concept reduces different durability models to a unique form what facilitates their comparison. Examples of the reduction are presented in the paper. The durability-based TNESF, described in this paper, is deduced from the durability functional $t^{*}(\sigma)$ but the former seems to be more robust in design applications and corresponding computer codes. Moreover, TNESF should be more convenient for identification from experimental data owing to its better properties (homogeneity in stress and monotonicity in time). Natural function classes for loading processes $\sigma_{i j}$ and properties of the TNESFs $\underline{\Lambda}$ on those classes are to be studied. Strength-based TNESFs (coinciding with the durability-based TNESFs at the points of continuity in time) and some methods of their direct interpolation along the durability diagrams under constant loading are supposed to be described in a separate paper. Methods for refinement of the TNESF identification (interpolation) from a finite number of experimental data need to be developed for an effective implementation of the concept in engineering practice. Adaptation of the identification ideas of [18] to the TNESFs looks promising. Expansion of approach 
of $[8,19]$ to non-local durability analysis for bodies with stress concentration is to be done. This approach can be also extended to fatigue strength analysis under cyclic [20,21] and non-cyclic loading.

Note also that the similar concept of the temporal normalized equivalent strain functional can be introduced by in same way by replacing successively the stress loading process $\sigma_{i j}(\tau)$ by the strain loading process $\varepsilon_{i j}(\tau)$ in the above reasoning.

\section{ACKNOWLEDGEMENT}

This work was completed under the research grant GR/M24592 "Non-local approach to high cyclic fatigue: Theoretical basis" of the Engineering and Physical Sciences Research Council, UK.

\section{Appendix}

\section{A Remarks on some damage measures}

Different damage measures are often introduced to reflect the material properties change under loading in comparison with a reference state. We present several of the most popular damage measures at an instant $t$ under the loading process $\sigma(\tau)$.

Geometrical damage measures (see $[1,3,7]$ :

$$
\begin{aligned}
\omega_{S}(\vec{n} ; \sigma ; t) & :=1-S(\sigma ; t) / S(0 ; 0) \Longrightarrow S(\sigma ; t)=\left(1-\omega_{S}(\vec{n} ; \sigma ; t)\right) S(0 ; 0) \\
\omega_{V}(\sigma ; t) & :=1-V(\sigma ; t) / V(0 ; 0) \Longrightarrow V(\sigma ; t)=\left(1-\omega_{V}(\sigma ; t)\right) V(0 ; 0) .
\end{aligned}
$$

Here $S(\sigma ; t)$ is a representative element net cross-section area with a normal vector $\vec{n}$ at an instant $t$, and $S(0 ; 0)$ is the corresponding area before loading; $V(\sigma ; t)$ is a representative element net volume at an instant $t$, and $V(0 ; 0)$ is the corresponding volume before loading.

Softening damage measure (see $[1,3,7]$ :

$$
\omega_{E}(\sigma ; t):=I-E(\sigma ; t) E^{-1}(0 ; 0) \Longrightarrow E(\sigma ; t)=\left(I-\omega_{E}(\sigma ; t)\right) E(0 ; 0)
$$

Here $E(\sigma ; t)$ is the (macro-) stiffness tensor at an instant $t$ and $E(0 ; 0)$ is the tensor before loading. Effective (micro-) stress tensor is taken as

$$
\tilde{\sigma}(t)=\left(I-\omega_{E}(\sigma ; t)\right)^{-1} \sigma(t)=E(0 ; 0) E^{-1}(\sigma ; t) \sigma(t) .
$$

Assuming that the damage is isotropic, it is often supposed that the geometric and softening damage measures coincide. General relations between the anisotropic softening and the geometrical damage measures for an elastic medium with cracks can be found in [22].

One of the main ideas of the continuum (softening) damage mechanics is an assumption (see [7] that all constitutive relations known for an undamaged material hold true also for 
the damaged material if one replaces there the macro-stresses $\sigma$ by the effective stresses $\tilde{\sigma}$. There exists also a temptation to use this idea for the strength prediction, that is, to write the strength condition for a damaged material in the form

$$
\Lambda_{00}\left(\left[I-\omega_{E}(\sigma ; t)\right]^{-1} \sigma(t)\right)<1
$$

if we know a strength condition $\Lambda_{00}(\sigma)<1$ for the virgin material. The problem however is that the function $\Lambda_{00}(\sigma)$ is principally unknown since the material at rupture is always damaged (not virgin). An exclusion can be the case when only aging damage (caused by non-mechanical reasons) is analysed. Another idea that the softening damage measure is a perfect strength indicator and one can write the strength condition in the form $F\left(\omega_{E}(\sigma ; t)\right)<1$ does also not always work: a "paradoxical" example when adding damage (crack array) increases strength is presented in [22, Section VII.A].

\section{B Strength absolute stability and durability diagram continuity}

Statement 5 The strength is absolutely stable under a process $\sigma_{i j}(\tau)$ at all $t<t^{*}(\sigma)$ if and only if the durability $t^{*}(\lambda \sigma)$ is a lower semi-continuous function of $\lambda$ at $\lambda=1$, that is, for any $\delta>0$ there exists $\epsilon(\delta)>0$ such that $t^{*}(\sigma)-t^{*}\left(\lambda^{\prime} \sigma\right)<\delta$ for any $\lambda^{\prime} \in(1-\epsilon, 1+\epsilon)$.

Proof. For any $\delta>0$ we denote $t_{\delta}=t^{*}(\sigma)-\delta<t^{*}(\sigma)$. Suppose strength is absolutely stable at all $t<t^{*}(\sigma)$ and particularly at the instant $t_{\delta}$. Then there exists $\epsilon(\delta)>0$ such that $t^{*}\left(\lambda^{\prime} \sigma\right)>t_{\delta}$ for all $\lambda^{\prime} \in(1-\epsilon, 1+\epsilon)$. Hence, $t^{*}(\sigma)-t^{*}\left(\lambda^{\prime} \sigma\right)<\delta$ for all $\lambda^{\prime} \in(1-\epsilon, 1+\epsilon)$ which proves the lower semi-continuity of $t^{*}(\lambda \sigma)$.

Conversely, let $t^{*}(\lambda \sigma)$ be lower semi-continuous in $\lambda$ at $\lambda=1$. Then for any $\delta>0$ there exists $\epsilon(\delta)>0$ such that $t^{*}(\sigma)-t^{*}\left(\lambda^{\prime} \sigma\right)<\delta$ for all $\lambda^{\prime} \in(1-\epsilon, 1+\epsilon)$. Hence for any $t<t^{*}(\sigma)$, we take $\delta(t)=t^{*}(\sigma)-t$ and have $t<t^{*}\left(\lambda^{\prime} \sigma\right)$ for all $\lambda^{\prime} \in(1-\epsilon, 1+\epsilon)$, what proves that the strength is absolutely stable under the process $\sigma_{i j}(\tau)$ at all $t<t^{*}(\sigma)$.

For MD processes, the lower semi-continuity of $t^{*}(\lambda \sigma)$ in $\lambda$ coincides with the right continuity of $t^{*}(\lambda \sigma)$ in $\lambda$ and we can reformulate the above statement in the form

Statement 6 The strength is absolutely stable under an MD process $\sigma_{i j}(\tau)$ at all $t<t^{*}(\sigma)$ if and only if the durability $t^{*}(\lambda \sigma)$ is a right continuous function of $\lambda$ at $\lambda=1$, that is, for any $\delta>0$ there exists $\lambda(\delta)>1$ such that $\left|t^{*}(\sigma)-t^{*}\left(\lambda^{\prime} \sigma\right)\right|<\delta$ for any $\lambda^{\prime} \in[1, \lambda]$.

\section{Proof of Remark 1}

We define $\underline{\lambda}_{s t}(\sigma ; t):=\sup \left\{\lambda: t_{s t}^{*}\left(\lambda^{\prime \prime} \sigma\right)>t \quad \forall \lambda^{\prime \prime} \in[0, \lambda]\right\}$. Since $t_{s t}^{*}\left(\lambda^{\prime \prime} \sigma\right) \leq t^{*}\left(\lambda^{\prime \prime} \sigma\right)$ then $\underline{\lambda}_{s t}(\sigma ; t) \leq \underline{\lambda}(\sigma ; t)$. 
Suppose $\underline{\lambda}_{s t}(\sigma ; t)<\underline{\lambda}(\sigma ; t)$. Then for any $\lambda_{0}$ such that $\underline{\lambda}_{s t}(\sigma ; t)<\lambda_{0}<\underline{\lambda}(\sigma ; t)$, we have,

$$
t_{s t}^{*}\left(\lambda_{0} \sigma\right) \leq t<t^{*}\left(\lambda_{0} \sigma\right)
$$

Consequently, strength is $\lambda$-unstable in any instant $t^{\prime} \in\left(t, t^{*}\left(\lambda_{0} \sigma\right)\right)$ under the process $\lambda_{0} \sigma$, that is, rupture appears at or before $t^{\prime}$ under the process $\lambda_{00} \sigma$ for any $\lambda_{00}>\lambda_{0}$. Thus, strength is $t$-unstable under the process $\lambda_{0} \sigma$ for any $\lambda_{0} \in\left(\underline{\lambda}_{s t}(\sigma ; t), \underline{\lambda}(\sigma ; t)\right)$ and hence $t^{*}\left(\lambda_{0} \sigma\right) \leq t$, and we arrive at a contradiction with the last inequality in (92). Thus $\underline{\lambda}_{s t}(\sigma ; t)=\underline{\lambda}(\sigma ; t)$.

\section{Proof of positive homogeneity for strength functionals}

Let $k>0$. Denoting $\tilde{\lambda}=k \lambda, \tilde{\lambda}^{\prime \prime}=k \lambda^{\prime \prime}$, we have from Definition 1 ,

$$
\begin{aligned}
& \underline{\lambda}(k \sigma ; t):=\sup \lambda:\left\{t^{*}\left(\lambda^{\prime \prime} k \sigma\right)>t \text { for all } \lambda^{\prime \prime} \in[0, \lambda]\right\} \\
= & \frac{1}{k} \sup (k \lambda):\left\{t^{*}\left(k \lambda^{\prime \prime} \sigma\right)>t \text { for all } k \lambda^{\prime \prime} \in[0, k \lambda]\right\} \\
= & \frac{1}{k} \sup \tilde{\lambda}:\left\{t^{*}\left(\tilde{\lambda}^{\prime \prime} \sigma\right)>t \text { for all } \tilde{\lambda}^{\prime \prime} \in[0, \tilde{\lambda}]\right\}=\frac{1}{k} \underline{\lambda}(\sigma ; t) .
\end{aligned}
$$

\section{E Proof of Statement 3}

Let $T$ be the supremum of $t$ such that (14) is satisfied. Suppose first $T<t_{s t}^{*}(\sigma) \leq \infty$. For any $t>T$, condition (14) is violated, that is $\underline{\lambda}(\sigma ; t) \leq 1$. Consequently, $t^{*}\left(\lambda^{\prime} \sigma\right) \leq t$ for any $\lambda^{\prime}>1$ and any $t>T$ due to the definition of $\underline{\lambda}(\sigma ; t)$ for MD materials. That is, $t^{*}\left(\lambda^{\prime} \sigma\right) \leq T<t_{s t}^{*}(\sigma)$ for any $\lambda^{\prime}>1$. However this contradicts to the definition of the critical time $t_{s t}^{*}(\sigma)$ since the strength appears to be $\lambda$-unstable at the instant $T<t_{s t}^{*}(\sigma)$ under the process $\sigma_{i j}(\tau)$. Consequently $T$ can not be less than $t_{s t}^{*}(\sigma)$.

Suppose now $t_{s t}^{*}(\sigma)<T \leq \infty$. Then we obtain from the definition of $T$ that condition (14) holds for any $t$ such that $t_{s t}^{*}(\sigma)<t<T$. Owing to Statement 1 , this implies $\lambda$-stable strength at the instant $t>t_{s t}^{*}(\sigma)$ under the process $\sigma_{i j}(\tau)$, which contradicts to the definition of the critical time $t_{s t}^{*}(\sigma)$. The contradiction proves that $T$ can not be greater than $t_{s t}^{*}(\sigma)$.

Hence $T=t_{s t}^{*}(\sigma)$.

\section{F Proof of Corollary 2}

Suppose first $t^{* *}=t_{s t}^{*}(\sigma)$. Then the right hand side of (15) follows from Statement 1 and the left hand side follows from Statement 3. 
Suppose now (15) is satisfied. Then $t_{s t}^{*}(\sigma) \geq t^{* *}$ owing to the left hand side of (15) and to Statement 3. But if $t_{s t}^{*}(\sigma)>t^{* *}$ then $\underline{\Lambda}\left(\sigma ; t^{* *}\right)<1$ owing to Statement 1 what contradicts to the right hand side of (15). Consequently $t^{* *}=t_{s t}^{*}(\sigma)$.

\section{G Proof of Statement 4}

Let $\underline{F}(\sigma ; t)=\infty$, then $\underline{\Lambda}(\sigma ; t)$ can not be zero since then Statement 1 implies strength and consequently $\underline{F}(\sigma ; t)<1$. On the other hand, $\underline{\Lambda}(\sigma ; t)$ can not be a finite number since then $\underline{\Lambda}(\sigma / C ; t)<1$ for any $C>\underline{\Lambda}(\sigma ; t))$, which means strength under the process $\sigma(\tau) / C$ and consequently $\underline{F}(\sigma / C ; t)<1$ and $\underline{F}(\sigma ; t)<C$.

Let $\underline{F}(\sigma ; t)$ is finite. The homogeneity of $\underline{F}(\sigma ; t)$ implies that any process $\sigma$ is monotonously damaging. Taking into account that $t<t^{*}(\lambda \sigma)$ implies $\underline{F}(\lambda \sigma ; t)<1$, we have from Definition $1 \mathrm{MD}$,

$$
\begin{aligned}
\underline{\lambda}(\sigma ; t) & =\sup \left\{\lambda>0: t<t^{*}(\lambda \sigma)\right\} \leq \sup \{\lambda>0: \underline{F}(\lambda \sigma ; t)<1\} \\
& =\sup \{\lambda>0: \underline{F}(\sigma ; t)<1 / \lambda\}=1 / \underline{F}(\sigma ; t) .
\end{aligned}
$$

This completes the proof of point (i).

Let now $\underline{F}(\sigma ; t)$ is right-continuous in the second argument at the considered time $t$. If $t^{*}(\lambda \sigma)$ is a durability under the process $\lambda \sigma$, then $\underline{F}\left(\lambda \sigma ; t^{*}(\lambda \sigma)\right) \geq 1$ since otherwise there exists $t>t^{*}(\lambda \sigma)$ such that $\underline{F}(\lambda \sigma ; t)<1$ due to the right-continuity and non-decreasing of $\underline{F}(\lambda \sigma ; t)$, which means $t^{*}(\lambda \sigma)$ is not the durability. Consequently, $\underline{F}(\lambda \sigma ; t) \geq 1$ if $t \geq t^{*}(\lambda \sigma)$. On the other hand, $t \geq t^{*}(\lambda \sigma)$ if $\underline{F}(\lambda \sigma ; t) \geq 1$ owing to the durability definition. Thus condition $t<t^{*}(\lambda \sigma)$ is equivalent to condition $\underline{F}(\lambda \sigma ; t)<1$.

Then we have from Definition $1 \mathrm{MD}$,

$$
\begin{aligned}
\underline{\lambda}(\sigma ; t) & =\sup \left\{\lambda: t<t^{*}(\lambda \sigma)\right\}=\sup \{\lambda: \underline{F}(\lambda \sigma ; t)<1\} \\
& =\sup \{\lambda: \underline{F}(\sigma ; t)<1 / \lambda\}=1 / \underline{F}(\sigma ; t) .
\end{aligned}
$$

Consequently, $\underline{\Lambda}(\sigma ; t)=1 / \underline{\lambda}(\sigma ; t)=\underline{F}(\sigma ; t)$. This completes the proof of point (ii).

\section{References}

[1] Kachanov, L.M.: Rupture time under creep conditions. Izv. Acad. Nauk SSSR, OTN, 8, 26-31 (1958).

[2] Rabotnov, Yu.N. On mechanism of long-time rupture. In: Strength Problems of Materials and Structures, pp. 5-7, USSR Academy of Sci. Publishing, 1959 (in Russian).

[3] Rabotnov, Yu.N. Creep Problems in Structural Members, Nauka, Moscow, 1966. English translation: North-Holland Publ., Amsterdam-London, 1969. 
[4] Kachanov, L.M. Introduction to Continuum Damage Mechanics. Martinus Nijhoff Publ. (Kluwer Academic Publ.), Dordrecht, 1986.

[5] Il'ushin, A.A.: About one theory of durability. Mech. of Solids (Izv. AN SSSR. MTT), 2(3), 21-35 (1967).

[6] Bolotin, V.V. Prediction of service life for machines and structures, ASME Press, New York, 1989.

[7] Lemaitre, J. A course of damage mechanics, 2nd edition, Springer, BerlinHeidelberg-New York, 1996.

[8] Mikhailov, S.E.: A functional approach to non-local strength conditions and fracture criteria - I. Body and point fracture. Engng Fracture Mech., 52, 731-743 (1995).

[9] Mikhailov, S.E. A functional approach to durability description: Basic functionals. In: Progress in Mechanical Behaviour of Materials, ICM8, Vol. III: Advance Materials and Modelling of Mechanical Behaviour, pp. 1210-1215, ed., Ellyin, F. \& Provan, J. W., Flemming Printing Ltd., Victoria BC, 1999.

[10] Nikiforovsky, V.S.: On kinetic character of brittle fracture of solids. Appl. Mech. and Techn. Phys., 18 (5), 150-157 (1976).

[11] Nikiforovsky, V.S. \& Shemyakin, E.I. Dynamic Fracture of Solids. Novosibirsk Univ. Press, Novosibirsk, 1979.

[12] Morozov, N. \& Petrov, Y. Dynamics of Fracture. Springer, Berlin-Heidelberg, 2000.

[13] Campbell, J.D.: The dynamic yielding of mild steel. Acta Matallurgica, 1, 706-710 (1953).

[14] Robinson, E.L.: Effect of temperature variation on creep strength of steels, Trans. ASME, 60 (1938).

[15] Robinson, E.L.: The effect of temperature variation on the long-time rupture strength of steels, Trans. ASME, 74, 777-780 (1952).

[16] Penny, R.K. \& Marriott, D.L. Design for Creep, McGraw-Hill, London, 1971.

[17] Hoff, N.J.: The necking and rupture of rods subjected to constant tensile loads. J. Appl. Mech., 46, (1953).

[18] Mikhailov, S.E. About interpolation of the non-local strength functionals by experimental data. In: 15th IMACS World Congress on Scientific Computations, Modelling and Applied Mathematics: Berlin, August 1997. Proceedings, vol. 3, pp. 515-520, ed., A.Sydow, Wissenschaft und Technik Verl., Berlin, 1997.

[19] Mikhailov, S.E.: A functional approach to non-local strength conditions and fracture criteria - II. Discrete fracture. Engng Fracture Mech., 52, 745-754 (1995). 
[20] Mikhailov S.E., Namestnikova I.V. Concept of Normalised Equivalent Stress Functionals for Cyclic Fatigue. Preprint PP/MAT/SEM/0102-005, Glasgow Caledonian University, 1-53 (2001).

[21] Mikhailov S.E., Namestnikova I.V. Non-local Approach to Cyclic Fatigue, Preprint PP/MAT/SEM/0102-006, Glasgow Caledonian University, 1-35 (2002).

[22] Kachanov, M.: Elastic solids with many cracks and related problems. Advances in Applied Mechanics, 30, 259-445 (1994). 


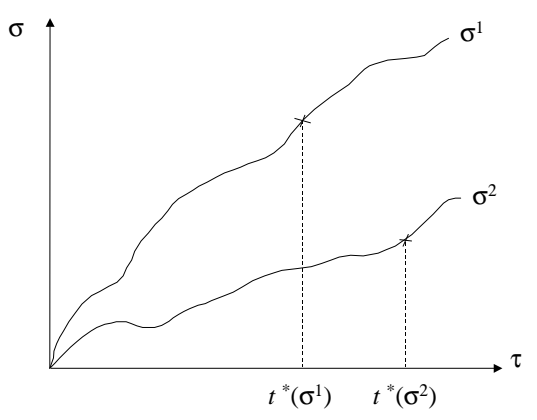

Figure 1: Loading processes and durabilities

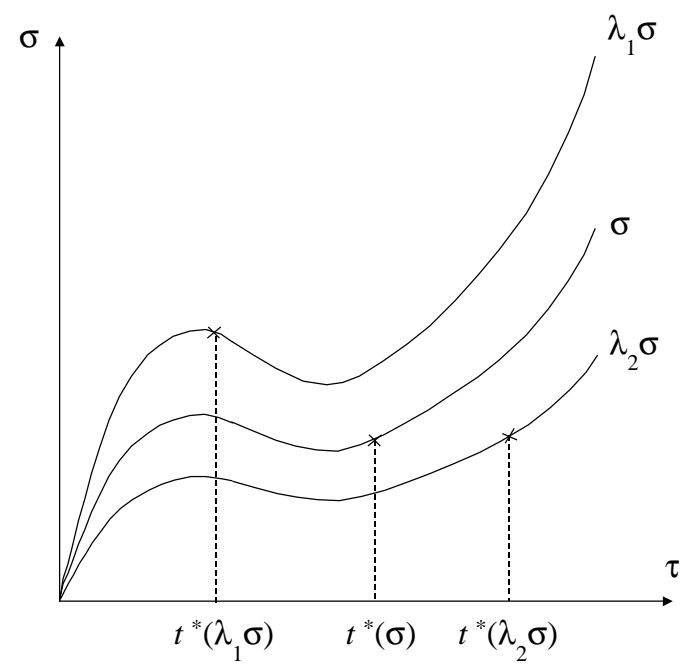

Figure 2: Proportional loading processes and durabilities, $0<\lambda_{2}<1<\lambda_{1}$. 
(a)

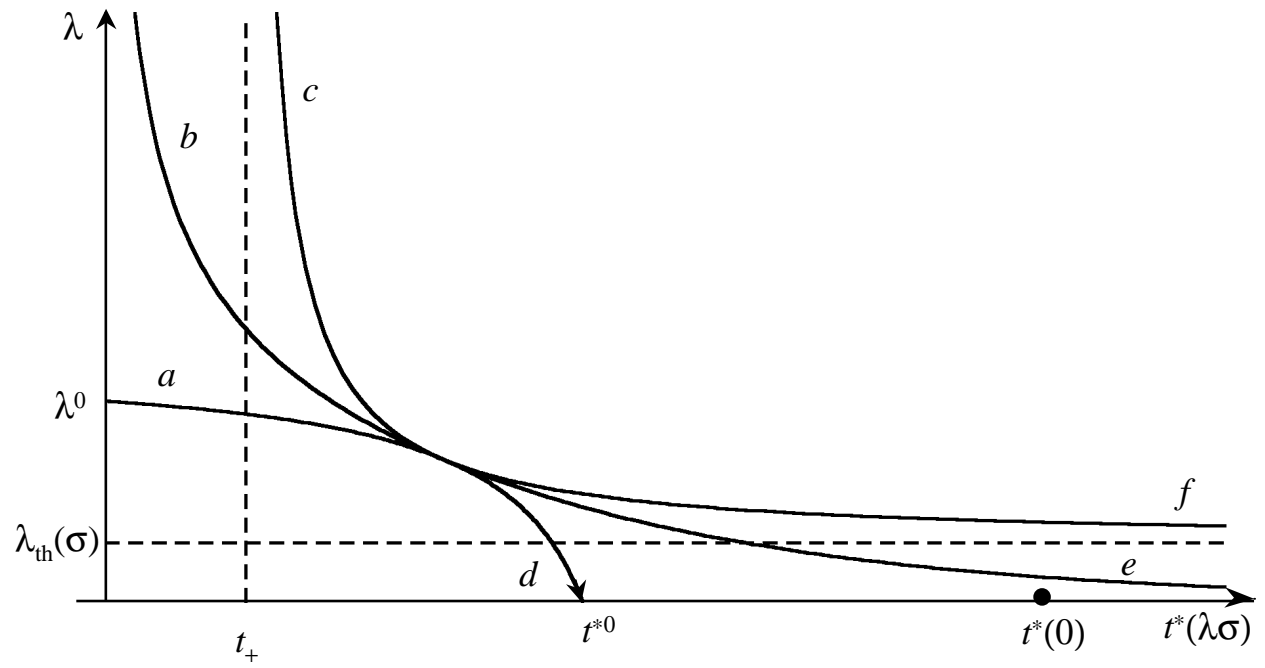

(b)

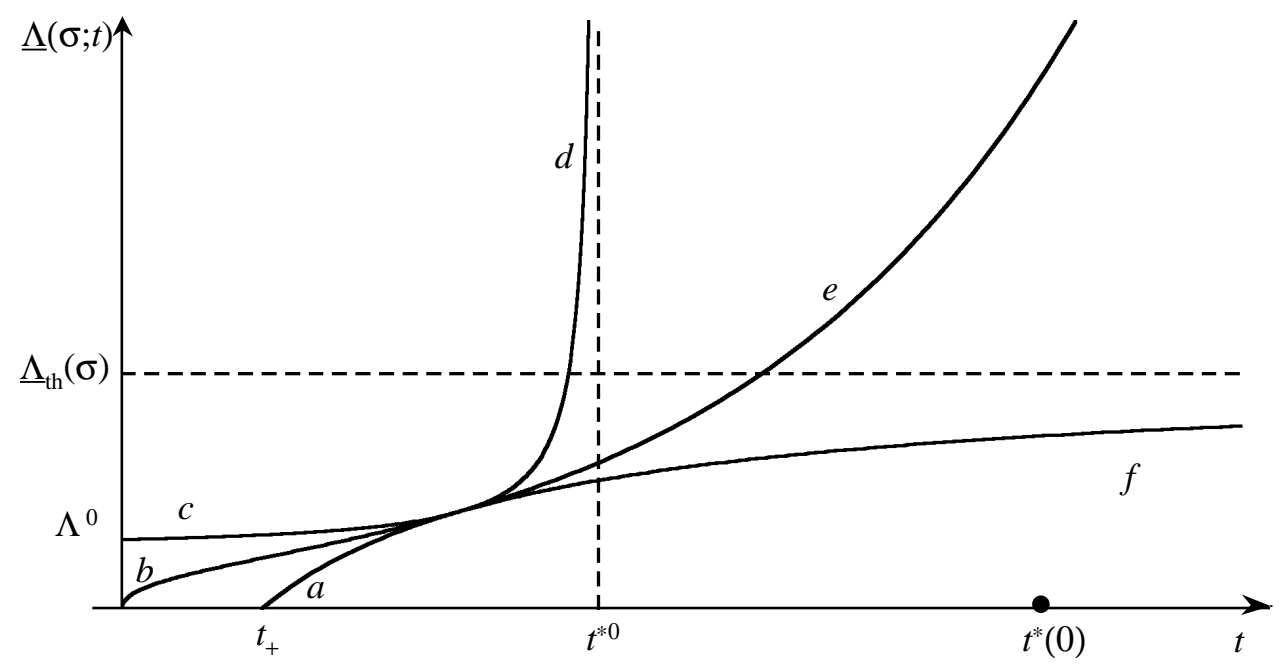

Figure 3: (a) Durability diagram for a process $\sigma_{i j}(\tau)$. (b) Normalized equivalent stress vs. $t$ for the process. 
(a)

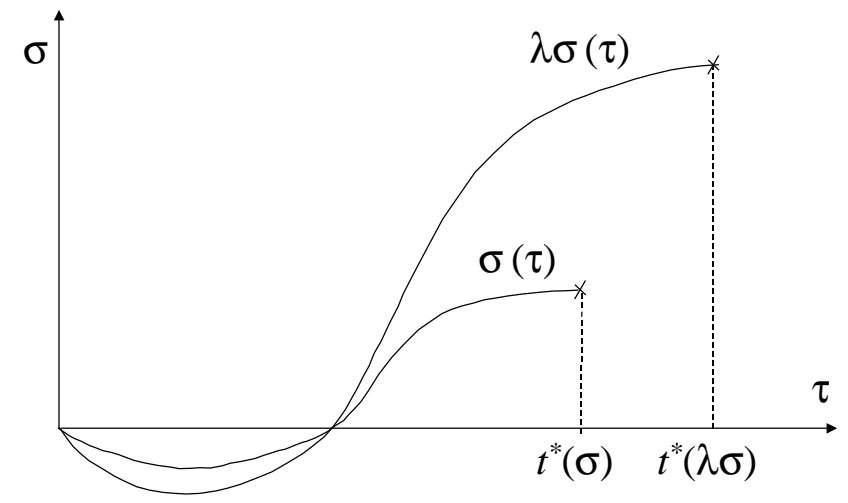

(b)
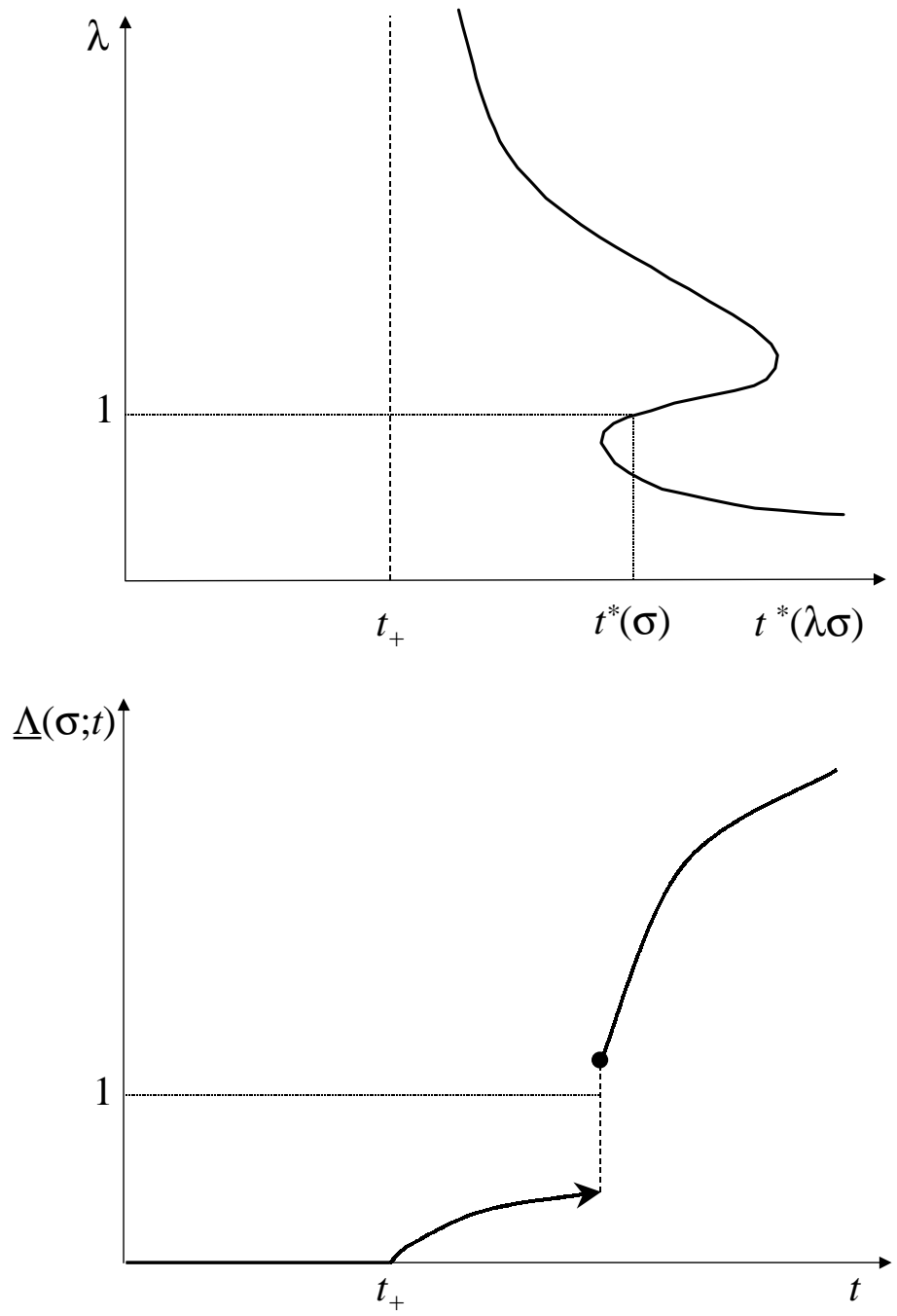

Figure 4: (a) Proportional non-monotonously damaging loading processes for $\lambda=1$ and $\lambda>1$. (b) Durability diagram for the process. (c) The normalized equivalent stress for the process. 
(a)

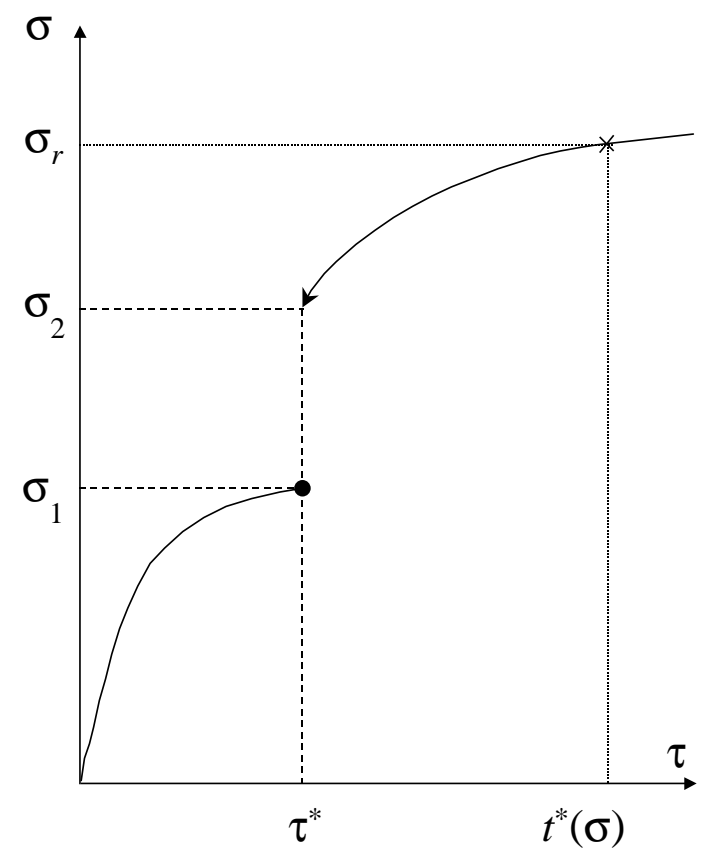

(b)

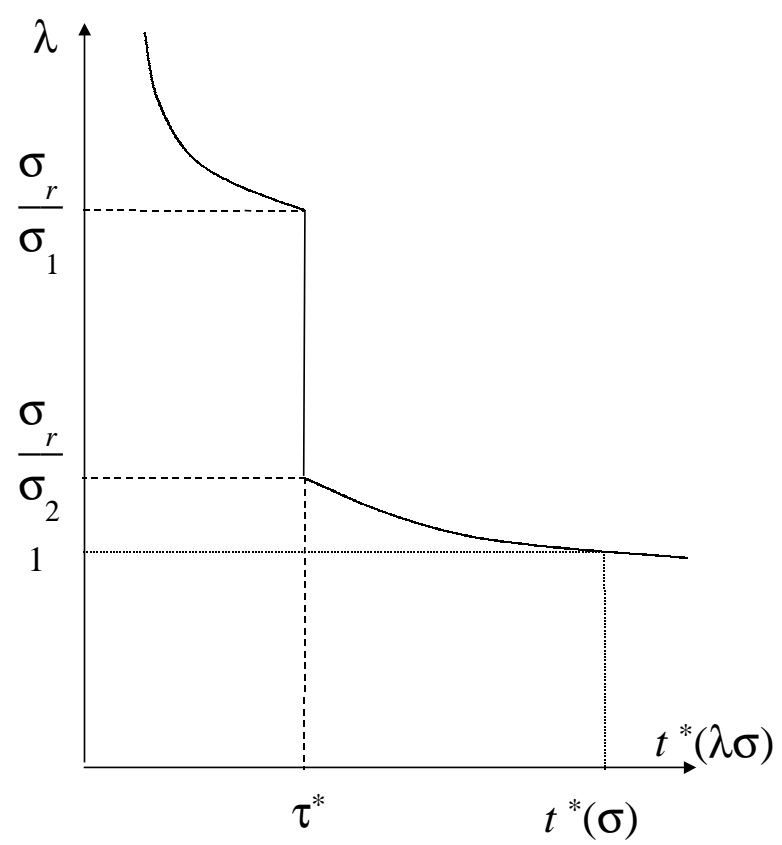

(c)

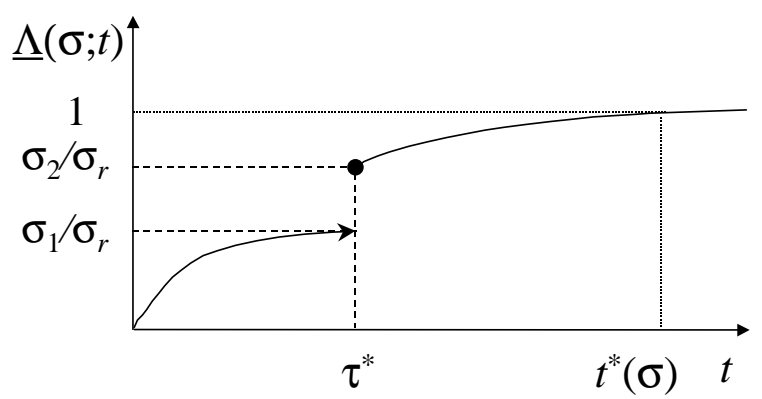

Figure 5: (a) Monotonous piecewise continuous loading process. (b) Piecewise continuous durability diagram generated by the process. (c) The normalized equivalent stress for the process. 
(a)

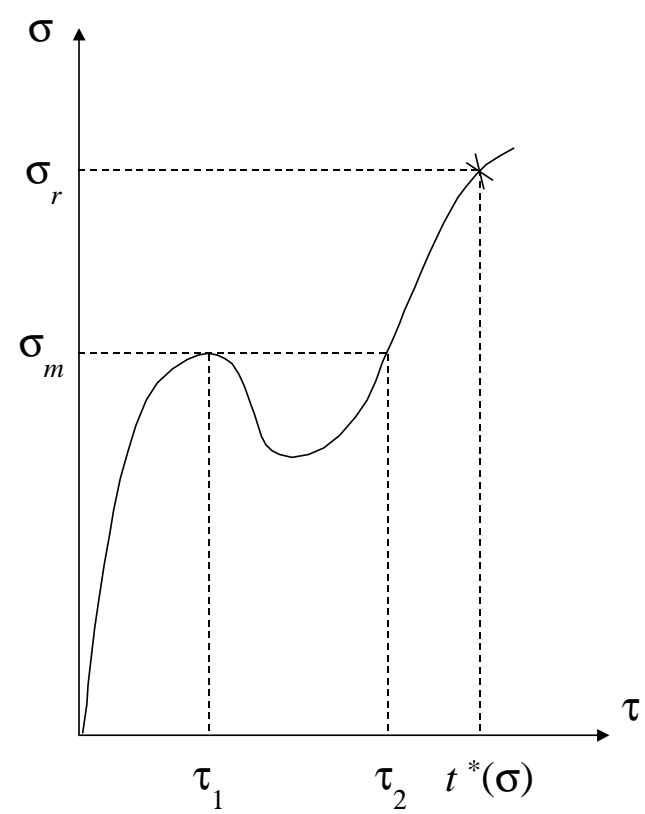

(b)

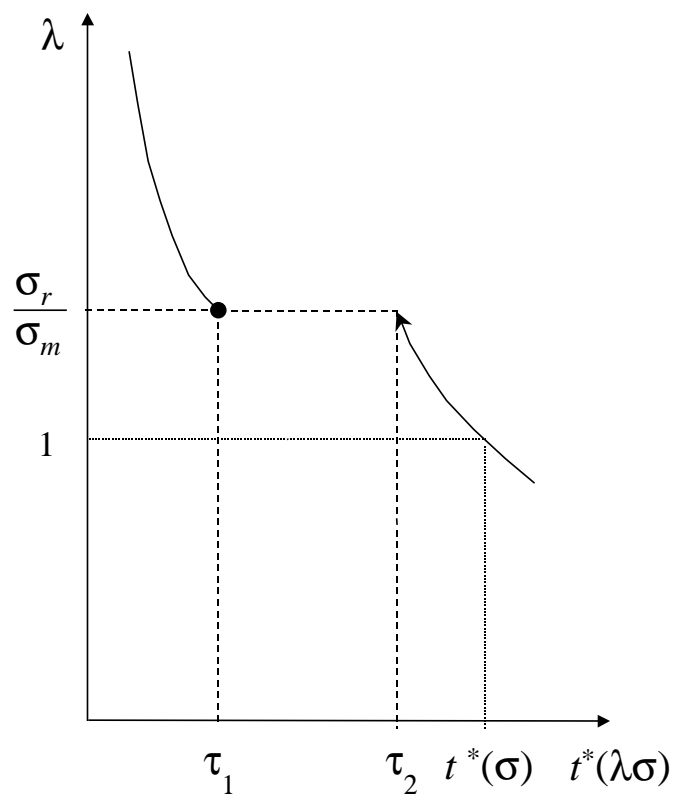

(c)

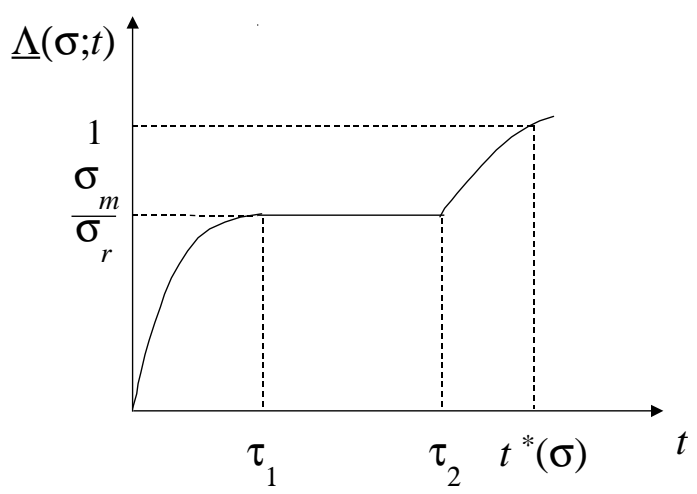

Figure 6: (a) Non-monotonous continuous loading process. (b) Piecewise continuous durability diagram generated by the process. (c) The normalized equivalent stress for the process. 
(a)

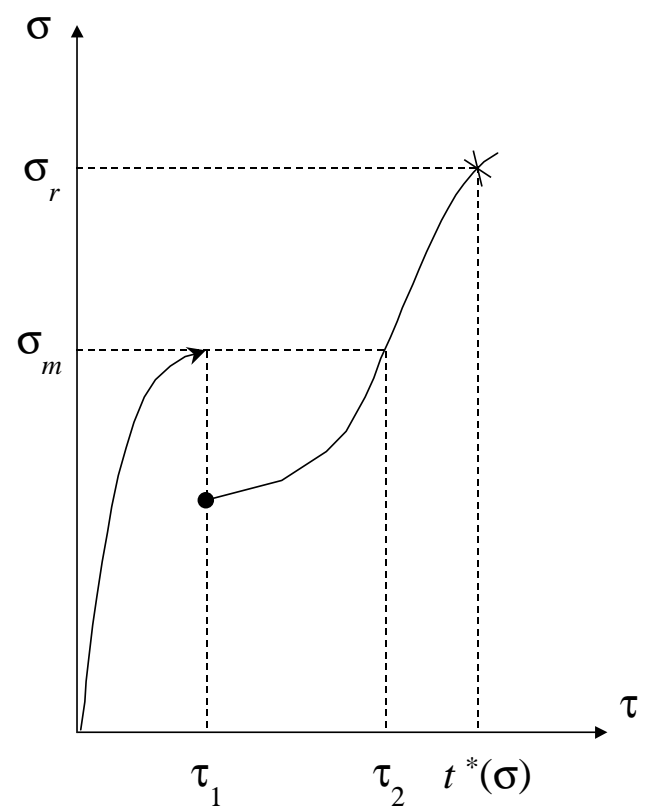

(b)

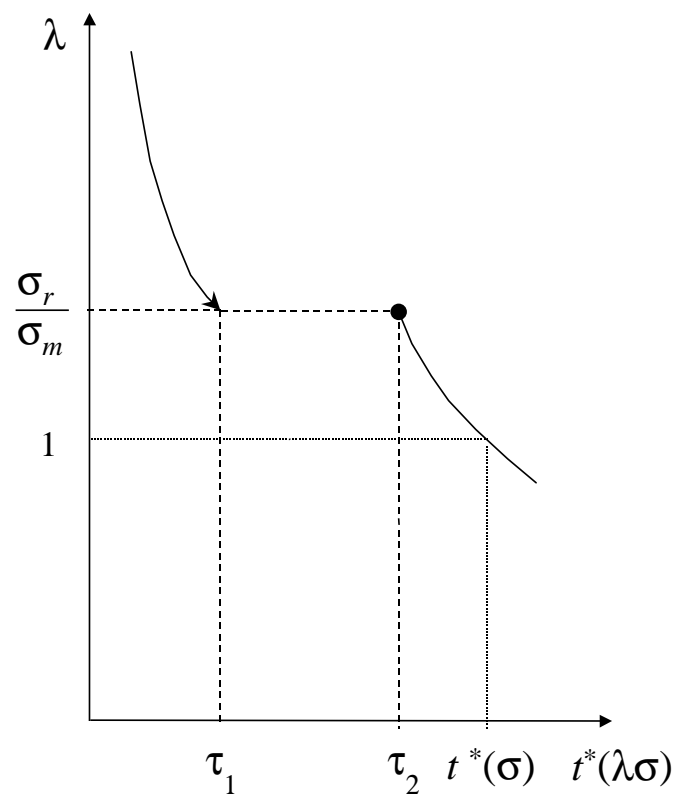

(c)

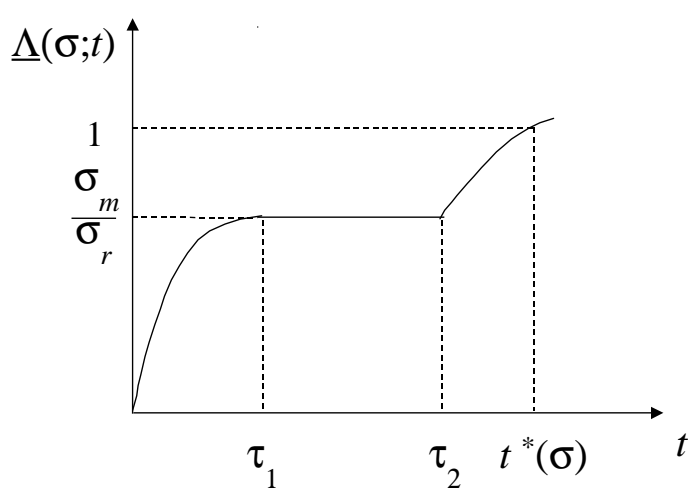

Figure 7: (a) Non-monotonous right-continuous loading process. (b) Piecewise continuous durability diagram generated by the process. (c) The normalized equivalent stress for the process. 